\title{
Examining the Influence of TBM-Ground Interaction on Electrical Resistivity Imaging ahead of the TBM
}

\author{
K. Schaeffer ${ }^{(1)}$, M.A. Mooney ${ }^{(1)}$ \\ ${ }^{(1)}$ Dept. of Civil \& Environmental Engineering, Colorado School of Mines, Golden, Colorado, USA \\ Corresponding Author: Kevin Schaeffer, kschaeff@mines.edu, (720)-933-7824
}

Keywords: tunnel boring machine, geoelectrics, electrical resistivity, geologic prediction

ABSTRACT:

Tunnel excavation by means of tunnel boring machines (TBMs) is susceptible to unknown changes ahead of the tunnel face. Geophysics offers a technique called electrical resistivity that can continuously, in real-time, spatially map the formation in front of the TBM. Electrical resistivity has been successfully established for many applications including vadoze zone hydrology, oil/gas location, mineral location and failure detection in geo-structures. Yet it has not been well-established for TBM excavations. This is in part due to the complexity of the TBM tunneling environment and the uncertain influence this environment may have on the success of TBM-integrated-electrical resistivity to predict changes ahead of the tunnel face. One significant uncertainty lies in the interface region that exists around the TBM created during the modification of the virgin formation by a mechanical mixing action of the rotating cutterhead and the injection of additives used to aid in the extraction of the muck and protect the cutting tools from frictional wear. In this study, we investigate the influence of this interface region on TBM-integrated-electrical resistivity for both hard rock and soft ground tunneling conditions through finite element modeling. Regarding the performance of TBM-integrated-electrical resistivity to detect changes ahead of the cutting face, the interface region holds significant influence for both earth pressure balance (EPB) and open mode tunneling conditions. Electrical resistivity for slurry based tunneling is not influenced by the interface region. Simulations suggest that TBM-integrated-electrical resistivity can be sensitive to a formation change that is located up to five diameters in front of the TBM.

\subsection{Introduction}

Tunnel boring machine (TBM) excavations are increasingly performed in urban environments (underneath critical buildings, beneath roadways and utilities, in close proximity to other tunnels and foundations) and in more complex geologies (mixed face conditions, water table, karstic rock etc.). Under such conditions, the excavation process incurs higher risk due to an unanticipated presence of ground changes, water bearing zones, weak regions or voids, and undocumented structural remnants (tie back anchors, abandoned wells, etc.). A considerable limitation in current TBM tunneling practice is the inability to characterize these unexpected changes ahead of the TBM. Methods that can continuously, and in real time, predict geologic changes and other hazardous conditions ahead of the TBM would provide significant risk reduction during excavation.

The field of geophysics offers non-destructive and continuous techniques that can potentially image ahead of the TBM without halting excavation like other more destructive methods such as probe hole drilling. At least two TBM-integrated-electrical resistivity systems are commercially available, the BEAM (Kaus \& Boening 2008) and the BEAM4 (Kopp 2012), and have been implemented on a few projects 
worldwide. Still, publications that document the underlying principles, implementation details, as well as strengths and limitations are scarce. Electrical resistivity has successfully been used in a multitude of applications including vadoze zone hydrology, oil and gas location, pollution detection and ore body mining (e.g., Mazac et al. 1987, Benson et al. 1997, Meju 2000, E.A. Atekwana 2000, Atekwana 2002). Given its success in other applications, is it unclear why electrical resistivity has not yet been fully developed and adopted universally in TBM tunneling.

The goal of this paper is to explore the fundamentals of TBM-integrated electrical resistivity and examine the complexities associated with TBM excavations and their influence on TBM-integrated electrical resistivity. Surface-based electrical resistivity is first presented to provide the fundamental basis for its application to the TBM tunneling environment. The paper then defines the important aspects of the TBM tunneling environment for both soft ground pressurized face and hard rock open mode conditions, particularly the TBM-ground interface and material properties within the interface. Six unique TBM-integrated-electrical resistivity electrode configurations are considered to determine how each configuration is influenced under different interface conditions. The paper will examine many material and geometric combinations for each of the TBM-integrated-electrical resistivity electrode configurations by using computational finite element modeling. This paper focuses on so-called DC resistivity methods and does not consider complex conductivity observed at higher AC frequencies through induced polarization (IP) methods. The extension to complex conductivity and IP is discussed in the paper.

\subsection{Electrical Resistivity Background}

An analysis of surface-based electrical resistivity provides the needed background for the TBM tunneling framework. A traditional surface electrode array is comprised of four electrodes $A, B, M$ and $N$ (Figure 1). $D C$ or very low frequency $(<1 \mathrm{~Hz}) A C$ current is injected between electrodes $A$ and $B$, and the resulting electric field (measured as an electrical potential in Volts) is measured between electrodes $M$ and N. Although higher frequency AC current injection has been adopted more recently to induce electrical polarization in geo-materials (e.g. Vinegar and Waxman 1984, Frye et al. 1998, Lesmes and Frye 2001, Binley et al. 2005, Revil and Florsch 2010), this study will focus on DC/low frequency AC only. Figure 1 illustrates a Wenner electrode configuration with a uniform electrode spacing of $a$. Over 102 unique surface electrode configurations have been established (e.g., Wenner, Schlumberger, Pole-Pole, Pole-Dipole, etc.) and are defined by the relative spacing and position between the four electrodes (e.g. Szalai and Szarka 2008). Each electrode configuration has strengths and weakness and so it is important to understand how the application environment (e.g. stratifications, presence of small anomalies, electrical noise, spatial constraints) could influence each array before choosing any specific array. In this study we will consider multiple arrays that are unique to TBM-integrated-electrical resistivity in order to observe how each array influences the predictive abilities of TBM-integrated-electrical resistivity.

The physics of electrical resistivity are macroscopically governed by Ohm's Law (Equation 1); the relationship between injected/retrieved current I (Amps) and measured electrical potential $V$ (Volts) is related to the electrical conductivity of the material $\sigma(\mathrm{S} / \mathrm{m})$ by the geometrical factor $K . K$ is quantified using the geometry of the electrode spacing (e.g. Binley and Kemna 2005, Kirsch and Yaramanci 2009). The literature often refers to electrical resistivity $\rho(\Omega \cdot \mathrm{m})$, which is the inverse of the electrical conductivity $\sigma$. The entire spectrum of geo-materials can display a significant range in $\sigma\left(10^{-16} \mathrm{~S} / \mathrm{m}\right.$ to $10^{7}$ S/m; Telford et al. 1990, Samouëlian et al. 2005, Reynolds 2011). 


$$
\sigma=\frac{I}{V * \boldsymbol{E}}
$$

In Figure 1, the magnitude of $I$ is set to $1 \mathrm{Amp}$ (electrode A) and -1 Amp (electrode B) for illustration. In practice, the magnitude of injected/retrieved current is user defined and is set based upon the scale, type of investigation, expected noise thresholds and expected contrast in electrical conductivity (Reynolds 2011). Current magnitude may be on the order of milliamps to 10 s of amps but is typically on the order of 10s of milliamps. Figure 1 shows the computational/analytical solutions for four different material property arrangements.

Figure 1 shows current flow lines that move from electrode $A$ to electrode $B$. Each current flow line shows a percentage of the total injected current passing above that particular line. For example, in Figure 1 (a) the $30 \%$ current flow line defines that $30 \%$ of the total current (in this case $30 \%$ of $1 \mathrm{Amp}=$ $300 \mathrm{~mA}$ ) flows above this line. The current flow lines are extracted from a mathematical model for a point source/sink in an infinite half space (e.g. Herman 2001). An important point about these models indicates that as depth increases $(z=a, 2 a, 3 a \ldots)$ the percentage of current becomes asymptotic - it would take an infinite depth $(z \rightarrow \infty)$ to account for $100 \%$ of the injected current.

Upon the introduction of electrical current, an induced electrical field is created and is measured as an electrical potential (measured in Volts) between electrodes $\mathrm{M}$ and $\mathrm{N}$; the induced electrical field is shown in Figure 1 with equipotential lines that are perpendicular to the current flow lines. The relationship between electrical equipotential lines and current flow lines is analogous to flow-nets that describe head loss during water seepage through geo-materials. Equipotential lines in Figure 1 are shown only to the extent between electrodes $M$ and $N$, but would normally exist past electrodes $M$ and N. 


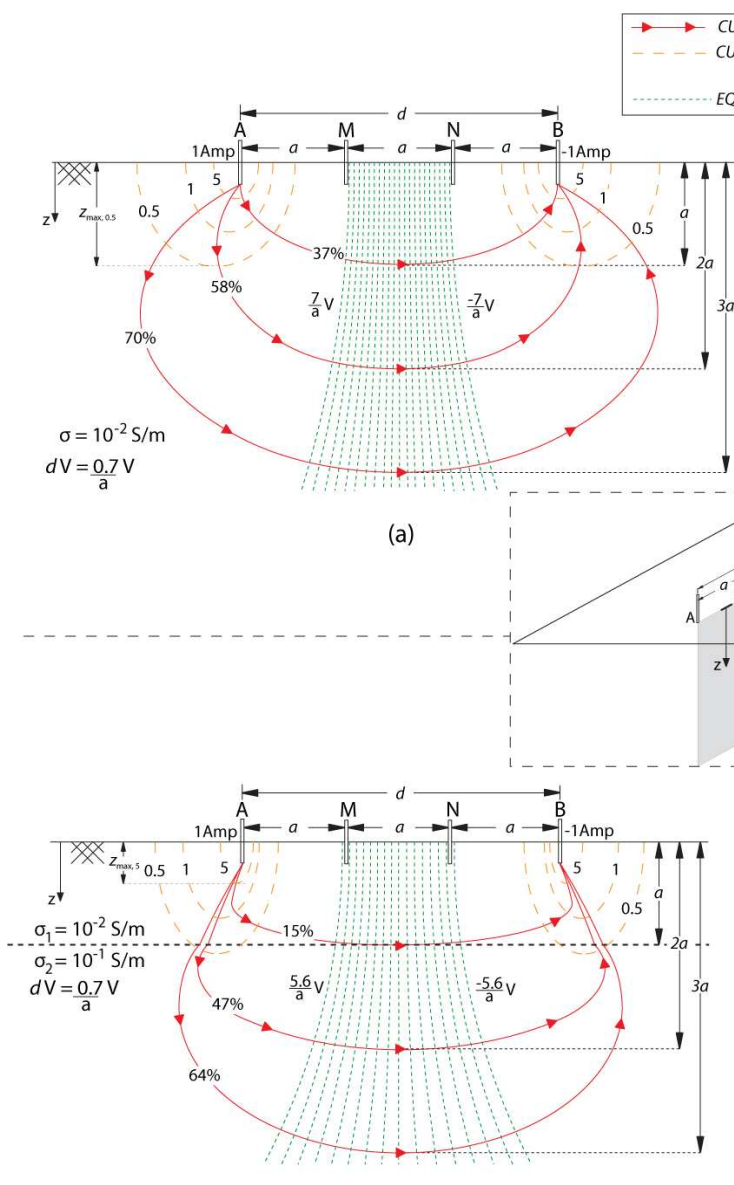

(c)

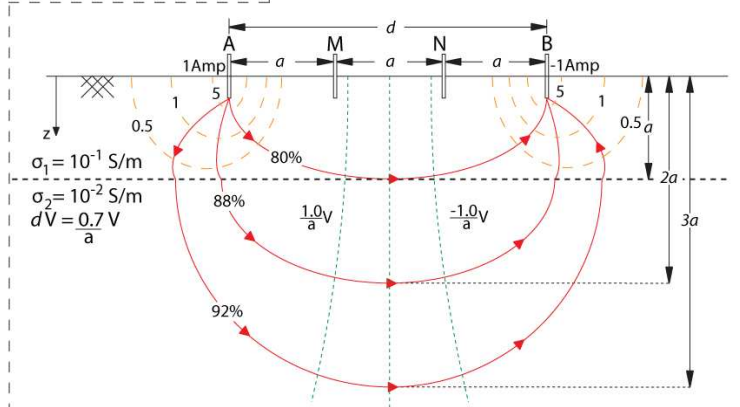

(d)

Figure 1: Slice of a three-dimensional, surface-based, electrical resistivity (DC) Wenner array. Current flow (\%), equipotential lines (V) and current density contours $\left(\mathrm{mA} / \mathrm{m}^{2}\right)$, for the electrical conductivity structure given by (a) homogeneous $\sigma=10^{-2} \mathrm{~S} / \mathrm{m}$, (b) homogeneous $\sigma=10^{-1} \mathrm{~S} / \mathrm{m}$, (c) infinite, horizontal planar difference for $\sigma_{1}=10^{-2} \mathrm{~S} / \mathrm{m}$ (top layer), and $\sigma_{2}=10^{-1} \mathrm{~S} / \mathrm{m}$ (bottom layer), and (d) infinite, horizontal planar difference for $\sigma_{1}=10^{-1} \mathrm{~S} / \mathrm{m}$ (top layer), and $\sigma_{2}=10^{-2} \mathrm{~S} / \mathrm{m}$ (bottom layer)

Figure 1(a) illustrates the case where the earth is assigned a homogeneous electrical conductivity, $\sigma=$ $10^{-2} \mathrm{~S} / \mathrm{m}$. The potential measured between $\mathrm{M}$ and $\mathrm{N}$ is $V_{M N} \approx \frac{14}{a} V$. In Figure $1(\mathrm{~b})$, the electrical conductivity of the earth is uniformly increased by a factor of 10 to the condition where $\sigma=10^{-1} \mathrm{~S} / \mathrm{m}$. In comparison to Figure 1(a), there exist no differences in the flow of electrical current through the earth. Since, the electrical conductivity is homogeneous in both cases, the path of current flow must remain identical as it has no alternative medium through which to flow. The distinction between Figures 1(a) and 1 (b) lies in $V_{M N}$. Due to the increase in electrical conductivity by magnitude of 10 , the potential decreases by the same magnitude according to Ohm's Law (Equation 1). The measured potential in Figure $1(\mathrm{~b})$ is $V_{M N} \approx \frac{1,4}{a} \mathrm{~V}$. The consequence in Figure $1(\mathrm{~b})$ is a decrease in resolution of the potential measurement and a decreased signal to noise ratio under the assumption of equal noise amplitudes.

Figures 1 (c) and 1 (d) show the cases where one homogeneous material overlays a different homogeneous material (e.g. a transition in geology or presence of groundwater). As shown, the planar 
difference occurs at a depth of $z=a$. The plane could exist at any depth and will be discussed later in this section. The first layered scenario is presented in Figure 1(c) where the top earth layer has a lower electrical conductivity than the bottom earth layer (e.g. sand on top of clay or unsaturated soil/rock overlying saturated soil/rock). The electrical current is more attracted to the bottom layer and current will penetrate to greater depth in order to reach the more conductive medium than for a completely homogeneous case (Figure 1(a)). In Figure 1(c), 15\% of the total current reaches the interface of the two layers as opposed to the $37 \%$ observed in Figures 1 (a) (or Figure 1(b)) at the same depth. Accordingly, a majority (85\%) of the current flows within the bottom layer. The electrical potential between electrodes $\mathrm{M}$ and $\mathrm{N}$ is marginally less than that for Figure 1 (a) $\left(V_{M N} \approx \frac{11}{a} V\right.$ in Figure 1 (c) compared to $V_{M N} \approx \frac{14}{a} V$ in Figure 1(a)). The presence of the conductive bottom layer reduces the measured potential because the electrical conductivity observed by the electrode array has increased on average when compared to the homogeneous case in Figure 1(a). By Ohm's Law, the measured potential must decrease given an increased electrical conductivity. As the difference moves closer to the surface the average conductivity will continue to increase, whereby the measured potential will continue to decrease. This result is shown in Figure 2 and is discussed later.

The second layered scenario is shown in Figure 1(d) where the top layer has a greater electrical conductivity than the bottom layer. The electrical current flow is mostly $(80 \%)$ confined to the conductive top layer. The potential between electrodes $\mathrm{M}$ and $\mathrm{N}$ here is slightly greater than Figure $1(\mathrm{~b})$ $\left(V_{M N} \approx \frac{2.0}{a} V\right.$ in $1 \mathrm{~d}$ compared to $V_{M N} \approx \frac{1.4}{a} V$ in $\left.1 \mathrm{~b}\right)$. Due to the presence of a resistive lower layer, the average electrical conductivity is decreased when compared to the homogeneous case in Figure $1(\mathrm{~b})$ and the measured potential must increase.

In reality, current flow lines do not exist and are only shown here to help illustrate the spatial distribution and direction of current flow. In three-dimensional space, the amount of current at any point of interest is represented by a current density given by the magnitude of current passing through a unit cross-sectional area. Mathematical models for percentage of current have been developed for simpler geometries like a horizontal planar difference shown in Figures 1 (c) and 1(d). When models are given more complex geometries and a range of electrical conductivities, analytical techniques that define percent current are not well established, and so, analysis may be administered by finite element modeling approaches that provide current densities rather than current percentages. Current density contour levels and associated maximum penetration distances of $z_{\max }$ are shown in Figure 1 for values of 5,1 and $0.5 \mathrm{~A} / \mathrm{m}^{2}$.

For a given depth of $z$, the percentage of the total injected current behaves similarly to $z_{\max }$. This is conveyed by the current density contour levels. For example, Figures 1 (a) and 1(b) show identical current percentages for any value of z. Likewise, current density contours and corresponding values for $z_{\max }$ are also identical between Figure 1(a) and Figure 1(b). When current penetrates deeper, as shown in Figure $1(\mathrm{c})$, the same current density contours also penetrate deeper and are shown with increased $z_{\max }$ values. Therefore, when quantifying the amount of current in a three dimensional space, this paper will utilize current density because it is analogous to percent current.

The cases shown in Figures 1(c) and 1(d) illustrate a horizontal planar difference at a depth of $z=a$. However, this planar difference could exist at any depth. Figure 2(a) shows $V_{M N}$ as a function of the 
depth of the planar difference. The results from both layered scenarios are presented; Figure 1 (c) and Figure $1(d)$. A bold, dashed line is shown for the scenario where the planar difference is located at a depth of $z=a$. Figure 2(a) shows how the measured potential changes as the planar difference is shifted with respect to the surface. As $z / a$ is increased, $V_{M N}$ tends to a constant value where the underlying layer has no influence on $V_{M N}$, and $V_{M N}$ equals the value of the corresponding homogeneous case (Figures $1(a)$ and $1(b))$. We refer to this as the far-field planar difference or condition $V_{\text {FAR FIELD. }}$

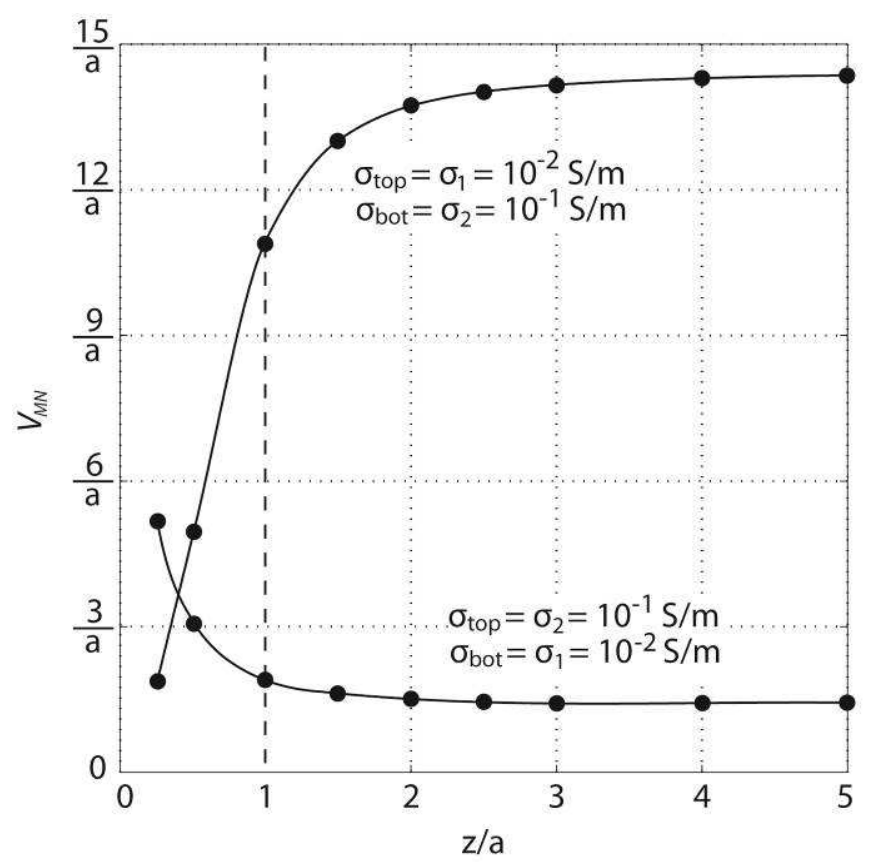

(a)

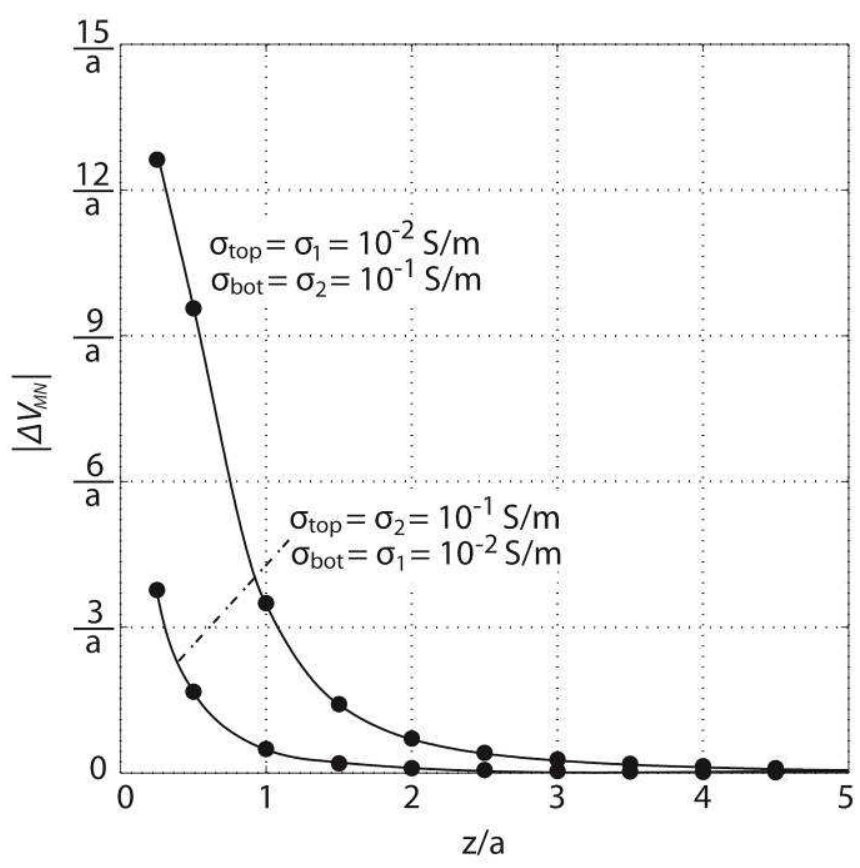

(b)

Figure 2: Surface-based, Wenner $(a=20 \mathrm{~m})$ electrical resistivity array, $(a)$ potential measurement, $V_{M N}$, for various depths, $\mathrm{z} / \mathrm{a}$, of the infinite, horizontal planar difference: $\sigma_{1}=10^{-2} \mathrm{~S} / \mathrm{m}, \sigma_{2}=10^{-1} \mathrm{~S} / \mathrm{m}$ and $\sigma_{1}$ $=10^{-1} \mathrm{~S} / \mathrm{m}, \sigma_{2}=10^{-2} \mathrm{~s} / \mathrm{m}(\mathrm{b})$ sensitivity curves $\left(V_{M N}-V_{\text {FAR FIELDD }}\right)$ for various depths, $z / a$, of the infinite, horizontal planar difference: $\sigma_{1}=10^{-2} \mathrm{~S} / \mathrm{m}, \sigma_{2}=10^{-1} \mathrm{~S} / \mathrm{m}$ and $\sigma_{1}=10^{-1} \mathrm{~S} / \mathrm{m}, \sigma_{2}=10^{-2} \mathrm{~S} / \mathrm{m}$

As the planar difference is moved closer to the surface $(z / a \rightarrow 0), V_{M N}$ changes exponentially. At some location of the planar difference, if $V_{M N}$ records a change in potential relative to the far-field condition, then the electrode array is considered sensitive to the planar difference for any distance less than or equal to its present $z / a$ location. Plots in the remainder of the paper present the change in potential ( $\Delta V$ $\left.=V_{M N}-V_{\text {FAR FIELD }}\right)$ as opposed to absolute measured potential. In Figure 2(b), for the condition where $\sigma_{1}=$ $10^{-2} \mathrm{~S} / \mathrm{m}, \sigma_{2}=10^{-1} \mathrm{~S} / \mathrm{m}$, the electrode array is considered sensitive at a depth less than or equal to $\mathrm{z} / \mathrm{a} \approx$ 4. In Figure 2(b), for the condition where $\sigma_{1}=10^{-1} \mathrm{~S} / \mathrm{m}, \sigma_{2}=10^{-2} \mathrm{~S} / \mathrm{m}$, the electrode array is considered sensitive at a depth less than or equal to $z / a \approx 2$. Therefore, the array is considered more sensitive to an incoming conductive layer than an incoming resistive layer. Computational analysis provides a noise free environment to observe these trends. In practice, electrical noise will be present and can affect the ability of an electrode array to detect differences like those shown in Figure 1. It is expected that electrical noise will limit the predication ability of electrical resistivity and is likely site specific regarding its magnitude/fluctuation. 


\subsection{TBM Tunneling Environment and the Interface Region}

The TBM tunneling environment is modeled with a hollow cylinder of diameter $D$ located at a cover depth of $C$ below the earth's surface (Figure 3(a)). The TBM structure is located at the closed end (righthand side in Figure 3) of the cylinder and is comprised of both the cutterhead and the shield. To the left of the TBM structure is a lining constructed of either shotcrete, cast-in-place concrete or precast concrete segments. Unlined tunnels are not considered here, but are of no consequence to the findings in this paper. The inside of the tunnel is comprised of air at atmospheric pressure. Figure 3(a) shows a simplified tunneling environment where the TBM makes perfect physical contact with the surrounding formation. In reality, there may exist some complexities regarding the perfect contact case shown in Figure 3(a) and may be generated by the introduction of cutting tools shown in Figures 3(b) and 3(c). These complexities are discussed in the following.

In tunnels where a precast lining is used, such as soft ground and unstable hard rock conditions, the TBM uses angled cutting tools (Figure $3(b)$ ) on the outer radius of the cutterhead to over-excavate and create an annulus around the shield that extends from the cutterhead to the back of the TBM shield. The material in the annulus depends highly upon the TBM type and will be discussed later in this section. Once the precast lining is constructed behind the TBM, the annulus between the outside (extrados) of the lining and the excavated opening is filled with a pressurized grout.

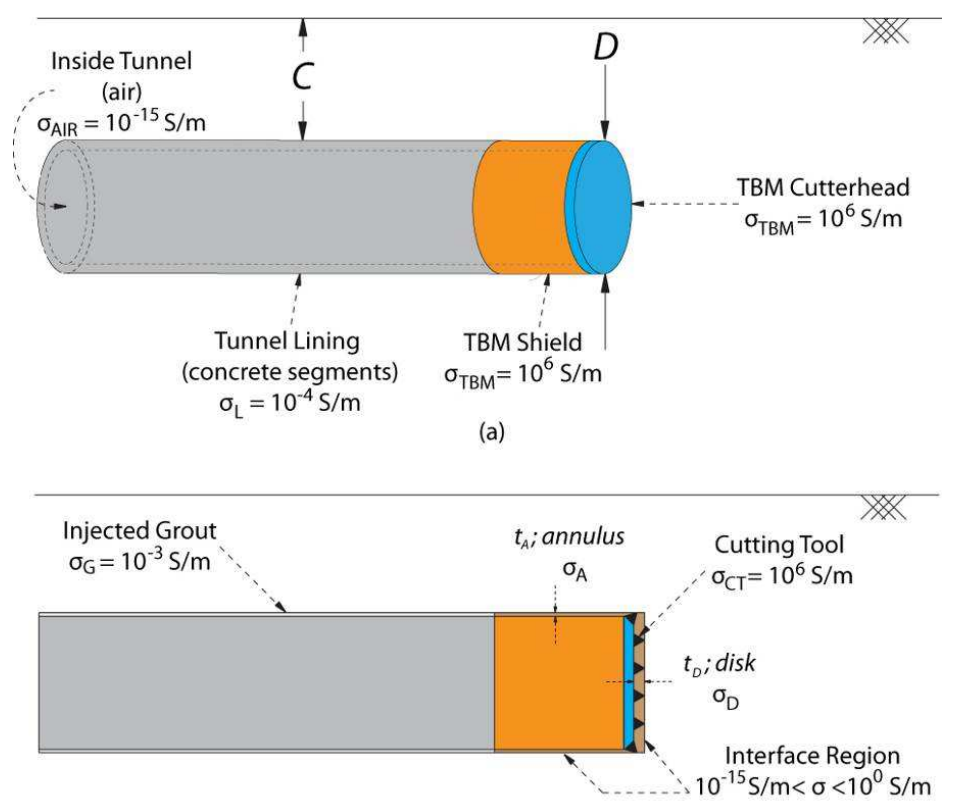

(b)

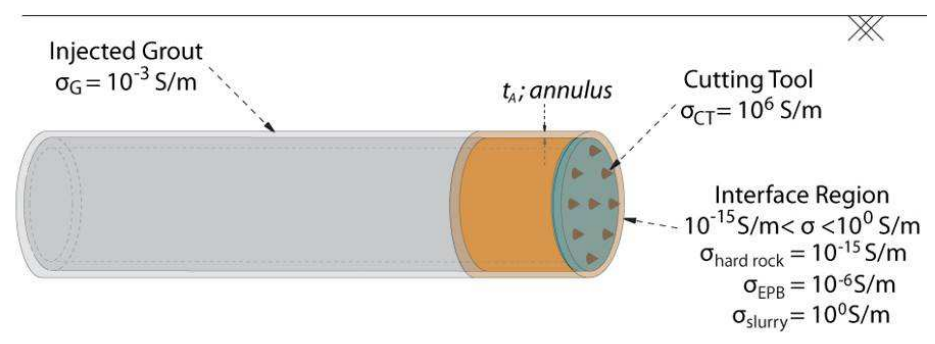




\section{Figure 3: TBM environment geometry and material definitions, (a) isometric simplified TBM,}

(b) side-view TBM with cutting tools, (c) isometric TBM with cutting tools

An interface region exists between the TBM and the formation, created by a combination of a) a mixing action from the cutting tools in front of the TBM cutterhead and b) an over-excavated annulus created from cutting tools on the outer radius of the TBM cutterhead. The dimensions and material properties of the interface region are quite variable and may depend on TBM type, cutting tool size and geometry, overburden pressure, ground type and additives injected during excavation.

Cutting tools include scrapers, rippers and/or disc cutters, each with a range of sizes. In general, cutting tool size can be on the order of $10-40 \mathrm{~cm}$ (e.g. Chang et al. 2006, Xue et al. 2009) and is specified based upon the diameter of the TBM, TBM type, and the ground conditions (soil type and overburden pressure). This paper will assume large cutting tools with a size of $40 \mathrm{~cm}$ in order to determine their maximum influence. Since the annulus is formed by means of angled cutting tools, this paper will assume an annulus thickness of $15 \mathrm{~cm}$. This annulus dimension is less than the dimension of the cutting tools themselves (due to their angled position) and is on the larger size as estimated by previous excavation practice (references). This study investigates the influence of the larger cutting tool size (i.e. larger interface region) because it aims to investigate the maximum influence of the interface region. For small cutting tools, the influence of the interface region would yield similar results but with less influence.

The electrical conductivity of the interface region could yield different values across TBM types and ground conditions. For hard rock tunneling, the region may be approximated as a volume of air with pieces of crushed and broken rock. The air phase will dominate in this case. For soft ground pressurized face tunneling, TBMs typically excavate using soil conditioning agents or water (earth pressure balance, EPB), or bentonite slurry (slurry TBM). To reflect these conditions, this study considers three values for the interface region conductivity based upon TBM type: $10^{-15} \mathrm{~S} / \mathrm{m}$ (hard rock TBM), $10^{-6} \mathrm{~S} / \mathrm{m}$ (EPB TBM), $10^{\circ} \mathrm{S} / \mathrm{m}$ (slurry TBM). The interface region is divided into a disk and annulus as illustrated in Figure 3. The disk (shown in Figure $3(\mathrm{~b})$ ) is approximated as a cylinder of thickness $t_{D}=40 \mathrm{~cm}$ (equal to the size of the cutting tool), and the diameter of the TBM, $D=10 \mathrm{~m}$. The disk is assigned an electrical conductivity, $\sigma_{D}$. The annulus (shown in Figures $3(\mathrm{~b})$ and $3(\mathrm{c})$ ) is approximated as a tube given an inner diameter equal to the TBM diameter, and an outer diameter equal to the TBM diameter plus $30 \mathrm{~cm}$. The annulus extends from the front of TBM cutterhead, back to the end of the TBM shield. The annulus is given an electrical conductivity $\sigma_{A}$.

Figure 4 shows four geometrical configurations of the interface region. Each of the configurations exists under the conditions shown adjacent to each configuration. Under highly complex conditions, it is possible where $\sigma_{D} \neq \sigma_{A}$ but this analysis will not consider these rare cases and always define that $\sigma_{D}=\sigma_{A}$. 


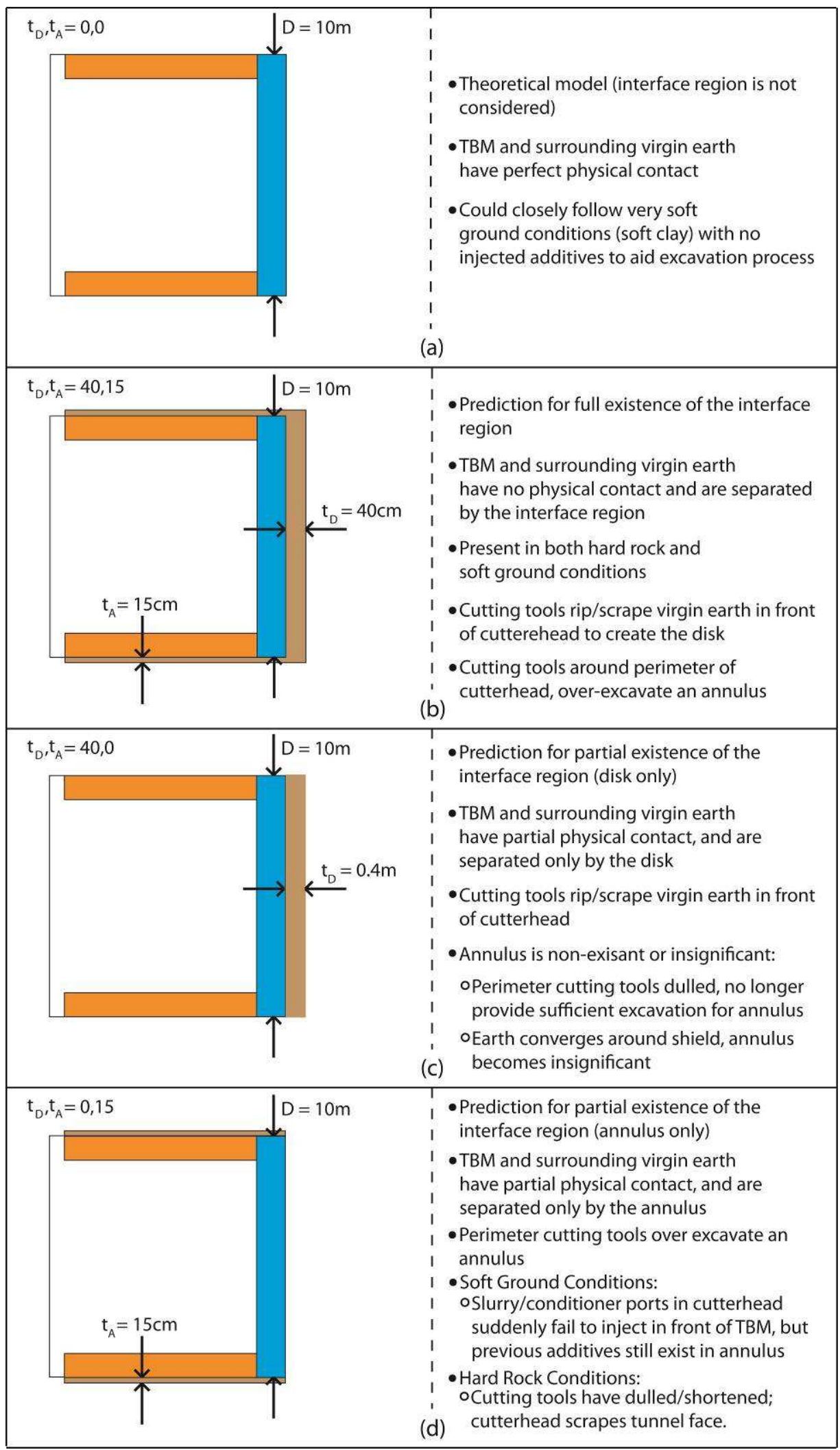

Figure 4: Four assumptions for the configurations for the Interface Region, (a) no region (b) disk and annulus (c) disk only (d) annulus only 
Figure 5 presents the six electrode configurations examined in this paper. Each illustration gives a qualitative representation of current flow lines. The flow lines, like those in Figure 1, move from the current source electrode $(A)$ to the current sink electrode $(B)$. Lines of equipotential are not shown here due to spatial constraints in the figure. Each of these electrode configurations will be analyzed for various assumptions of the interface region from Figure 4 (geometry, material property). The evaluation of each of the electrode arrays will be carried out by comparing observed current densities and measured potential sensitivity. A total current of $15 \mathrm{~A}$ is injected into the formation and is based upon analysis performed in Schaeffer (2014) for safe levels of current injection/retrieval. This value is theoretical; a case by case analysis of allowable current injection is required for practical implementation.

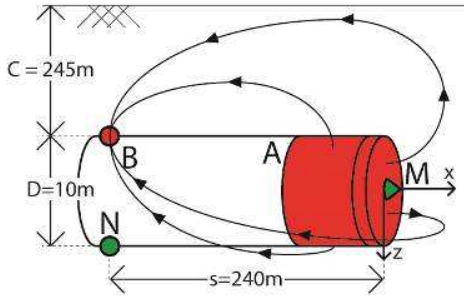

(a)

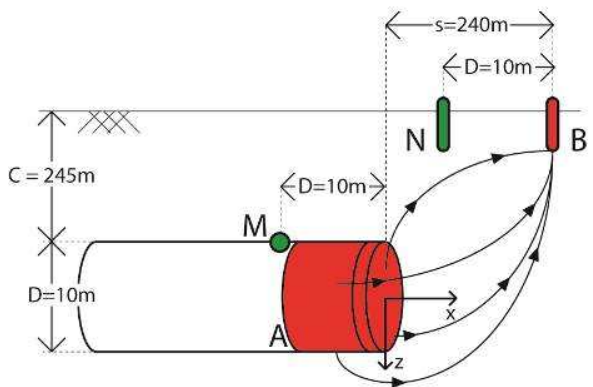

(b)

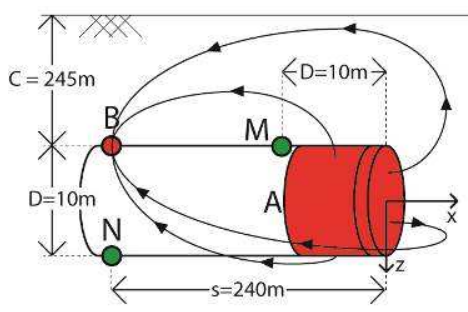

(c)

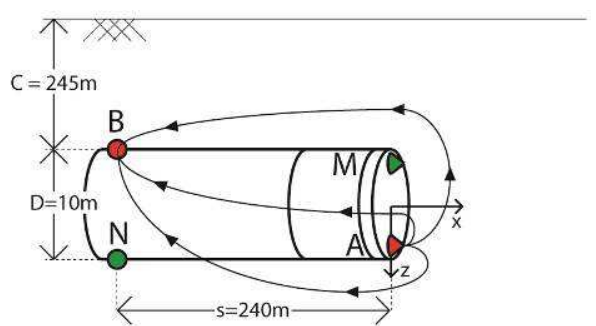

(d)

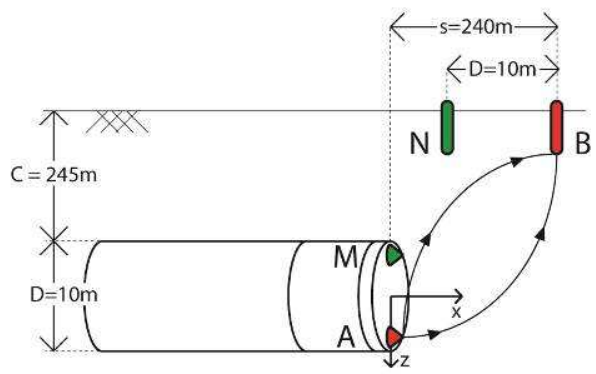

(e)

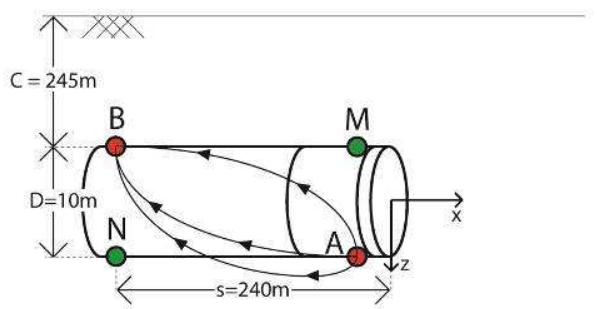

(f)

Figure 5: Six unique electrode arrays for TBM-integrated electrical resistivity with qualitative current flow lines

This paper will examine the influence of the interface region on each of the electrode arrays presented in Figure 5. Since it is unclear as to the most advantageous electrode array, this paper breaks up the analysis into two parts by examining the current injection/retrieval electrodes ( $A$ and $B$ ) and the electrical potential measuring electrodes ( $\mathrm{M}$ and $\mathrm{N})$, separately, under the same interface region 
conditions. To do this, this analysis will rely on two types of ground conditions similar to those presented in Figure 1. Figure 6 shows the ground conditions used for the analysis in this paper.

In Figure 6(a), the TBM is operating in a homogeneous earth condition where the electrical conductivity of the earth is assigned a value of $\sigma$. This condition is used to evaluate each model combination (TBM type, interface region assumption, and electrode configuration) for maximum current density penetration distance (consistent with $z_{\max }$ in Figure 1 ). The direction of interest is horizontal $(x)$ and in the direction of excavation. $x_{\max }$ is used to define maximum current penetration distance.In Figure $6(b)$, the TBM is surrounded in a homogeneous earth condition $\sigma_{1}$ and is approaching a homogeneous material $\sigma_{2}$ at a distance $x / D$ ahead of the TBM. The planar difference is moved closer to the TBM and the sensitivity of the potential measuring electrodes is observed for each model combination (both electrode configuration and assumption for the interface region).

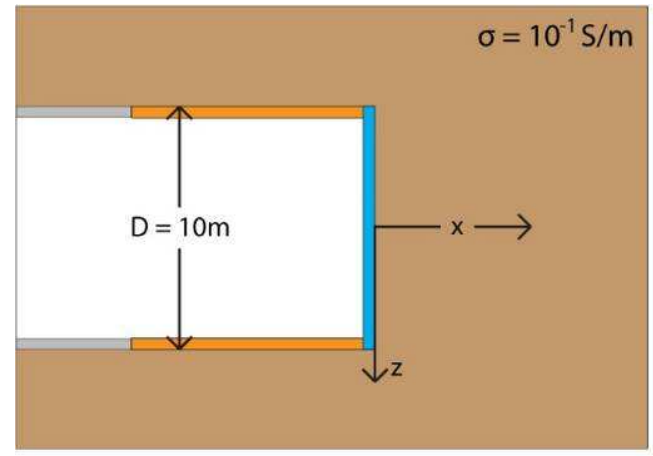

(a)

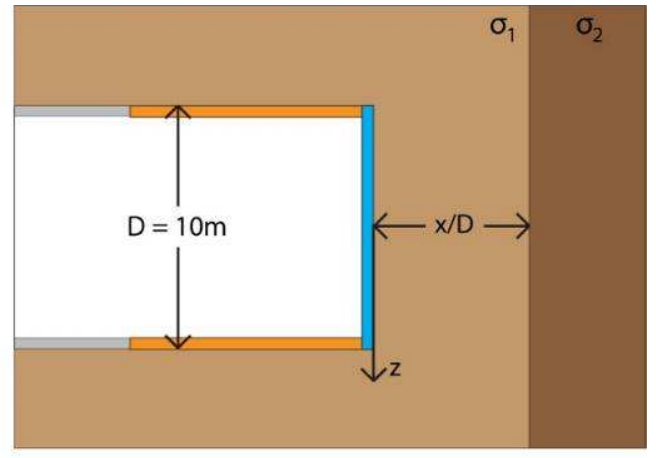

(b)

Figure 6: (a) TBM in a homogeneous ground condition, (b) TBM in a homogeneous ground condition, $\sigma_{1}$, approaching a homogeneous infinite vertical planar difference, $\sigma_{2}$

\subsection{Finite Element Model}

Finite element models were developed to perform the analysis in the following sections. The models use the COMSOL Multiphysics finite element software. COMSOL solves physics based partial differential equations to observe physical phenomenon in three dimensional space. COMSOL's AC/DC module simulates the flow of electrical flow through three dimensional space (e.g. surfaces, volumes, points) given user defined geometry, electrical material properties (namely electrical conductivity), and current injection/retrieval.

The front and center of the TBM cutterhead is located in the middle of a $500 \mathrm{~m}$ length cube $(x, y, z)=(0,0,0)$. The $10 \mathrm{~m}$ diameter TBM is located at a cover $C=245 \mathrm{~m}(245 \mathrm{~m}+10 \mathrm{~m} / 2=250 \mathrm{~m})$. The shield has a length equal to $D$. To simulate an infinite domain, COMSOL's infinite elements feature was used on five of the six sides of the model, where one side (ground surface) was left unchanged in order to simulate realistic boundary effects.

Figure 7(b) illustrates a region of electrical insulation between the cutting tools and the cutterhead. For electrode array that utilize individual cutting tools for either current inject or potential measurement, the tools must be electrically isolated from any other metallic (highly conductive) part of the TBM body. 
The consequence of not isolating these components would result in a short circuit. The thickness of the insulation is set to $0.05 \mathrm{~m}$ but its size is inconsequential to the modeling results.

Tetrahedral elements of a minimum size $0.2 \mathrm{~m}$ and a maximum size $3 \mathrm{~m}$ were used to mesh the TBM (shield, cutterhead, tunnel lining etc.). Tetrahedral elements of minimum size $3 \mathrm{~m}$ and maximum size $17.5 \mathrm{~m}$ were used for the earth around the TBM, with the exception of the "area of interest" (AOI; shown in Figure 7). The AOI is a rectangular prism located directly in front of the TBM and extends to the far right side of the model $(x>0)$. The AOI is created to provide a finer mesh in front of the TBM in order to mitigate coarse mesh averaging close to the electrode array. The AOI is dimensioned $30 \mathrm{~m}$ by $30 \mathrm{~m}$ by $250 \mathrm{~m}$ and is given minimum and maximum tetrahedral element sizes of $0.1 \mathrm{~m}$ and $3 \mathrm{~m}$, respectively.

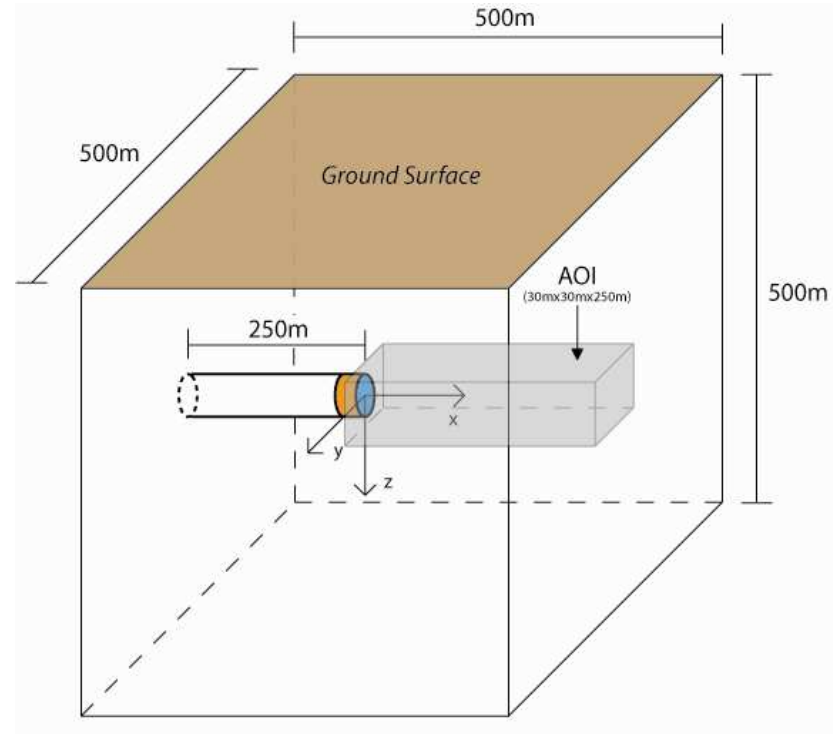

(a)

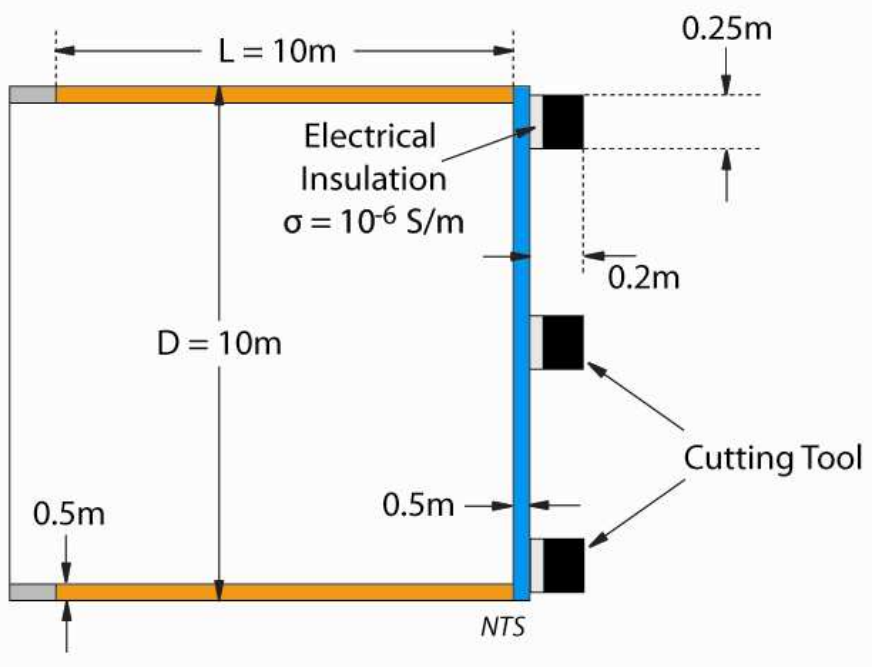

(b)

Figure 7: Finite element domain and dimensions (a) with TBM and refined element mesh Area of Interest (AOI), (b) close-up cross section of TBM with cutting tools

\subsection{Results}

\subsection{Current Density Analysis}

Current density contour plots convey how well each electrode array can inject current ahead of the TBM for each of the various interface region geometries and material types. Figure 8 shows contour plots of current density $\left(\mathrm{mA} / \mathrm{m}^{2}\right)$ on the vertical $x$ - $z$ plane $(y=0)$ around the TBM cutterhead for the electrode configuration shown in the center of the plots (configuration from Figure 5(a)) and for hard rock tunneling where the interface region is air $\left(\sigma_{A}=\sigma_{D}=10^{-15} \mathrm{~S} / \mathrm{m}\right)$. Each of the four contour plots in Figure 8 corresponds to each assumption regarding the presence of the interface region, as defined in Figure 4. Current density contours of $10,7.5,5,2.5$, and $1 \mathrm{~mA} / \mathrm{m}^{2}$ are shown. For reference, the average current densities observed within the formation, just outside the interface region, are shown in Figure 8. 

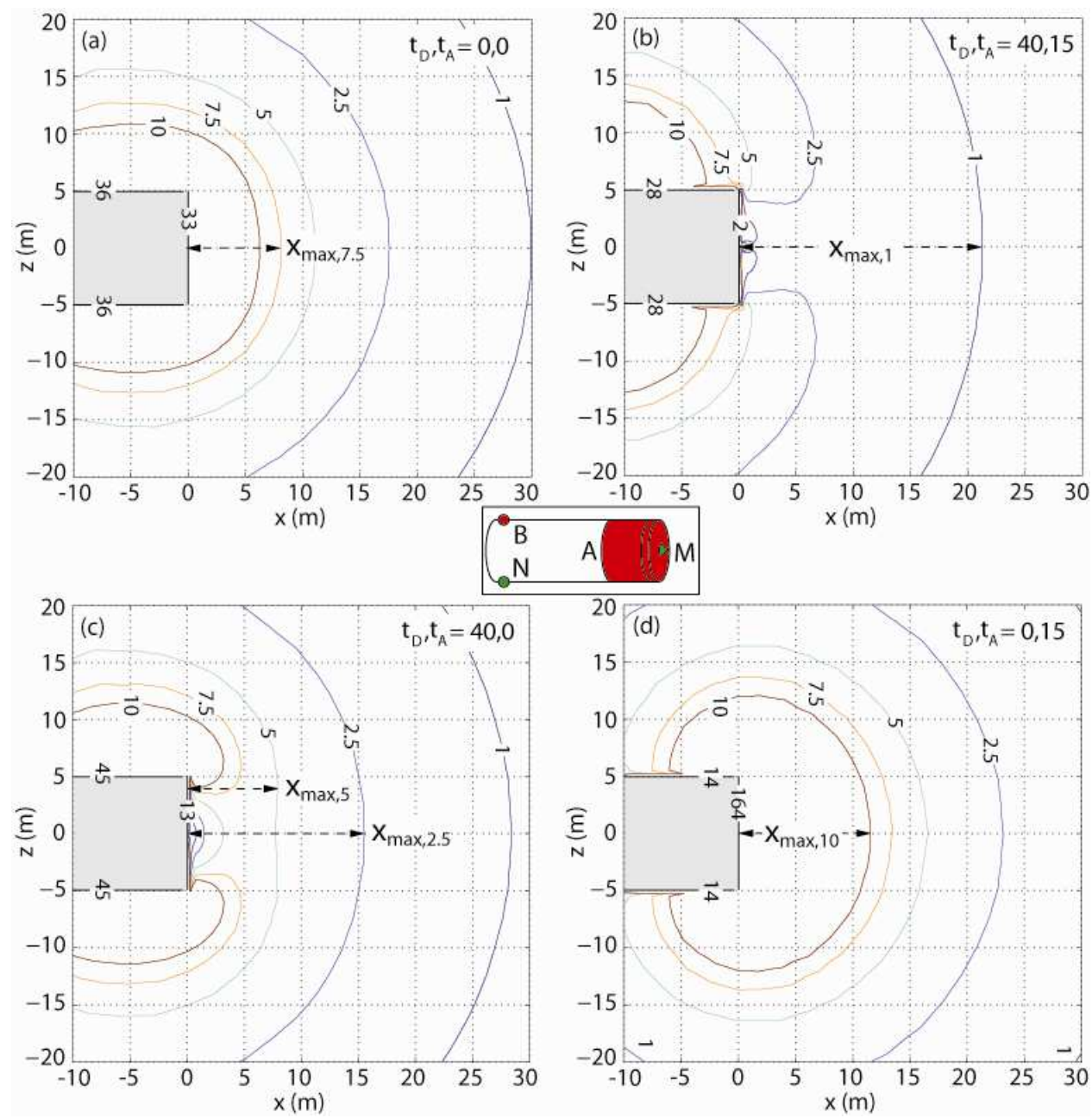

Figure 8: Current density $\left(\mathrm{mA} / \mathrm{m}^{2}\right)$ contour plots for electrode configuration Figure $1(\mathrm{a}), x(\mathrm{~m})$ vs. $z(\mathrm{~m})$, around the TBM showing maximum penetration distance, $x_{\max }(\mathrm{m})$, for current density contour levels: $10,7.5,5,2.5,1 \mathrm{~mA} / \mathrm{m}^{2}$. All contour plots shown for homogeneous earth condition (Figure 6(a)), and hard rock tunneling $\left(\sigma_{A}=\sigma_{D}=10^{-15} \mathrm{~S} / \mathrm{m}\right)$. Assumptions for the Interface Region configuration as shown in Figure 4: (a) no region, (b) disc and annulus, (c) only disc, (d), only annulus

Figure 8(a) illustrates the current density field for the baseline case where the TBM cutterhead and shield are in direct contact with the ground (i.e. no interface region). In this baseline case, current propagates into the ground in a spherical manner around the TBM. As current propagates from its source (in this case the cutterhead and shield), current density decays with $x$ due to geometrical spreading. The current density of $33 \mathrm{~mA} / \mathrm{m}^{2}$ at the cutterhead decays to $7.5 \mathrm{~mA} \mathrm{~mA} / \mathrm{m}^{2}$ at $x \approx 8 \mathrm{~m}$ due to geometrical spreading.

As shown in Figures 8(b), 8(c) and 8(d), the presence of an air-filled interface region has a significant influence on the distribution of current density around the TBM. In contrast to the baseline case, which distributes current density somewhat evenly around the TBM (due to perfect TBM contact with the formation), cases (b), (c) and (d) distribute current density in a way that is dependent upon the distribution of the interface region. For example in case (b), the interface region is comprised of both the disk and the annulus. Even though both the disk and the annulus are present, the annulus is given a thickness that is much smaller than the disk. Therefore, the annulus provides a path of lesser resistance 
due to its smaller thickness ( $15 \mathrm{~cm}$ annulus compared to $40 \mathrm{~cm}$ disk) and more current density is seen concentrated around the annulus. In this case, prediction ahead of the TBM is reduced because current does not flow as far out in front of the TBM in the $x$-direction. If the annulus was given a thickness equal to the disk $\left(t_{A}=t_{D}\right)$, then current would be observed more equally concentrated as the baseline case. For cases (c) and (d), the behavior follows the same logic regarding the dependence of current density distribution on the distribution of the interface region. The case in Figure 8(d) shows the greatest current density penetration distance because current is focused through the TBM cutterhead.

One aspect that is not clear from the figure pertains to current densities observed outside of the interface region in case (b). In general, these are much lower than for any of the other cases. This is due to a phenomenon not displayed in the subplot. As a result of the interface region entirely encapsulating the outer TBM surface, the path of least resistance is through the back on the TBM and through the grouted region $\left(\sigma=10^{-3} \mathrm{~S} / \mathrm{m}\right)$ just outside the tunnel lining. Only a fraction of the total injected current escapes through the interface region, and therefore, lower current densities are observed.

In general, if more current can escape out of the disk (as opposed to the annulus or through the grouted lining), then greater values in $x_{\max }$ can be observed. $x_{\max }$ and current density contour $\left(\mathrm{mA} / \mathrm{m}^{2}\right)$ information in Figure 8 are presented in Figure 9(a). The results from all four plots in Figure 8 are collapsed into Figure $9(\mathrm{a})$ to show the relative influence of each of the interface region conditions on the electrode array and TBM type (hard rock, slurry and EPB). Figure 9(a) confirms the discussion above, where $t_{D}, t_{A}=0,15$ provides the highest current density penetration and $t_{D}, t_{A}=40,15$ provides the least current density penetration.

The rest of Figure 9 shows the results from simulations using the five remaining electrode configurations from Figure 5. Each of the other five electrode configurations are subjected to the four interface region $t_{D}, t_{A}$ combinations and are shown in their respective subplots. Their associated current density data, like Figure 8 , are collapsed into plots that compare current density to $x_{\max }$. Figure 9 presents simulation results for hard rock and EPB tunneling (i.e. their plots overlay one another). This is because the differences in influence between the highly resistive air in hard rock tunneling $\left(\sigma_{A}=\sigma_{D}=10^{-15} \mathrm{~S} / \mathrm{m}\right)$ and foam conditioned soil in EPB tunneling $\left(\sigma_{A}=\sigma_{D}=10^{-6} \mathrm{~S} / \mathrm{m}\right)$ were negligible. 

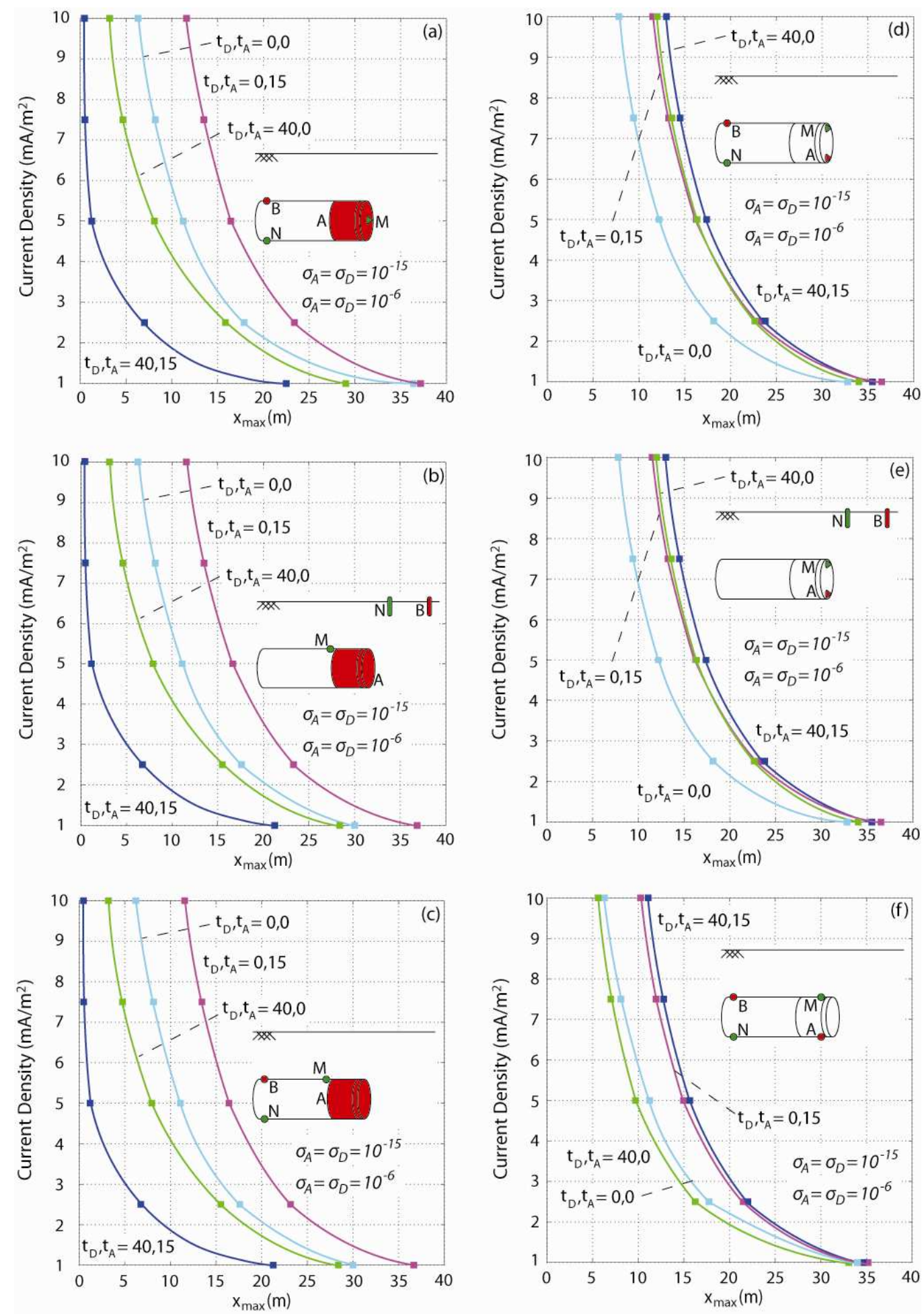

Figure 9: Maximum penetration distance, $x_{\max }(\mathrm{m})$, of current density $\left(\mathrm{mA} / \mathrm{m}^{2}\right)$ in front of cutterhead $(x>0)$ for hard rock and EPB tunneling, (a) Figure 5(a) electrode configuration, (b) Figure 5(b) electrode configuration, (c) Figure 5(c) electrode configuration, (d) Figure 5(d) electrode configuration, (e) Figure 5(e) electrode configuration, (f) Figure 5(f) electrode configuration 
Figure 9 shows that current density decays as $x_{\max }$ is increased and is explained by the geometrical spreading observed from the contour plots in Figure 8. Regardless of electrode array or interface region scenario, each curve has relatively the same shape of decay. The difference between each curve is a horizontal shift in $x_{\max }$ that is dependent upon the proportion of current density leaving through the disk compared to the annulus. After current escapes the interface region, current must flow through the same homogeneous formation and the current flow behavior must be the same outside of the interface region.

For open mode (hard rock) and EPB TBM tunneling, current density propagation is influenced more for electrode configurations that inject current from the entire TBM cutterhead and shield (Figure 9(a), 9(b) and $9(\mathrm{c})$ ) because they allow for such large areas of the current source $(A)$ to be isolated from the formation by the interface region. Electrode configurations that inject current from a single isolated cutting tool allow for less influence as the cutting tool has a smaller surface area to influence, is electrically isolated from the TBM, and always has at least partial contact with the formation. In comparison to configurations that inject through the entire cutterhead and shield, cutting tool injected relies on the electrical insulation of the TBM by the interface region. If the interface region is not entirely present (i.e. full electrical isolation of the TBM from the formation), then current is drawn back to the highly conductive TBM.

Figure 10 provides values of $x_{\max }$ for a range in current density (from 1 to $10 \mathrm{~mA} / \mathrm{m}^{2}$ ) for slurry TBM tunneling where the interface region $=\sigma_{A}=\sigma_{D}=10^{\circ} \mathrm{S} / \mathrm{m}$. In contrast to the hard rock and EPB models, the slurry TBM models assign an interface region electrical conductivity that is greater than that of the formation (here by one order of magnitude). Even though one order of magnitude is significant, the interface region thickness is not large enough to influence the results for a slurry TBM. In these cases, all four assumptions for the interface region tend to overlap one another or are otherwise very closely spaced. In comparison to hard rock and EPB TBMs, Figure 10 suggests that $x_{\max }$ is not significantly influenced by the electrode array or assumption for the interface region. For slurry tunneling, the ability to propagate current density rests in the range of hard rock and EPB tunneling where the case of $t_{D}, t_{A}=$ 0,0 for hard rock or EPB tunneling is consistent with values observed for slurry tunneling. 

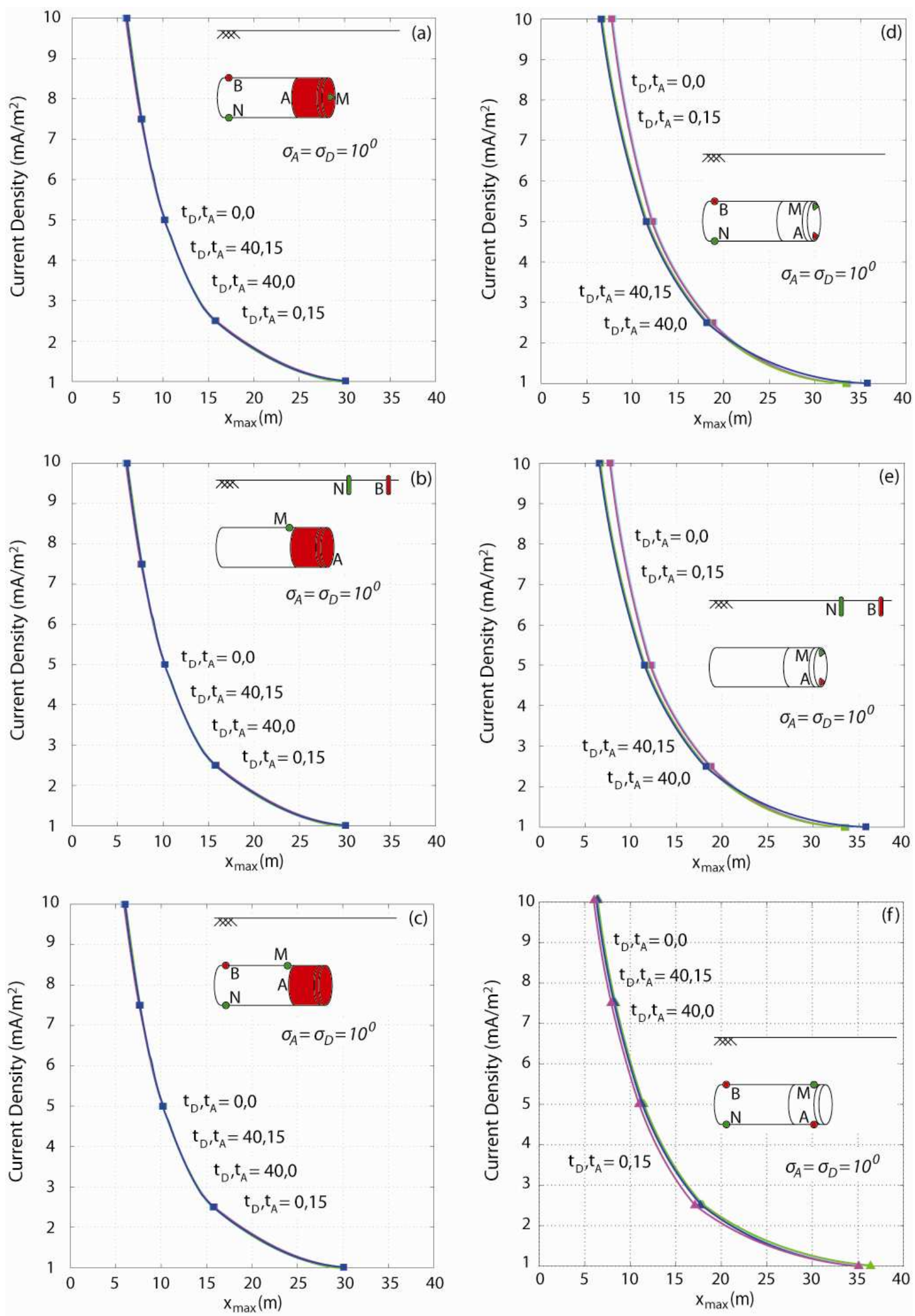
Figure 10: Maximum penetration distance, $x_{\max }(\mathrm{m})$, of current density $\left(\mathrm{mA} / \mathrm{m}^{2}\right)$ seen in front of TBM cutterhead $(x>0)$ for slurry shield tunneling, (a) Figure 5(a) electrode configuration, (b) Figure 5(b) electrode configuration, (c) Figure 5(c) electrode configuration, (d) Figure 5(d) electrode configuration, (e) Figure 5(e) electrode configuration, (f) Figure 5(f) electrode configuration

\subsection{Potential Measurement Sensitivity Analysis}

In this section we analyze the influence of the interface region on the electrical potential measuring electrodes $\mathrm{M}$ and $\mathrm{N}$, particularly the sensitivity of the potential measurement to a vertical planar difference ahead of the TBM cutterhead. In reality, there exist many forms of geologic differences including boulders, karstic voids - water or air filled, seams, faults or water bearing regions. These geologic differences are not explicitly considered here.

Figures 11 through 14 present the change in measured potential $\Delta V_{M N}$ as the TBM approaches a vertical planar difference in the formation ahead of the TBM. In these plots, the change in potential $\Delta V_{M N}$ is with respect to far-field condition, i.e., $V_{M N}-V_{\text {FAR FIELD. }} \Delta V_{M N}$ vs. $x / D$ is shown for each interface region configuration (e.g. $t_{D}, t_{A}=40,15$ ) and material property (e.g. $\sigma_{A}=\sigma_{D}=10^{-15} \mathrm{~S} / \mathrm{m}$ ). Sub-plot (a) presents the condition where the formation in which the TBM resides has $\sigma_{1}=10^{-2} \mathrm{~S} / \mathrm{m}$, and the formation at a distance $x / D$ in front of the TBM has $\sigma_{2}=10^{-1} \mathrm{~S} / \mathrm{m}$. Sub-plot (b) presents the reverse condition.

In Figures 11a through $14 a, \Delta V_{M N}$ becomes increasingly negative as $x / D$ decreases. This result is due to the approaching planar difference that is more conductive than the formation around the TBM. As the TBM advances toward to the planar difference, the observed electrical conductivity around the TBM continues to increase (i.e., current is more attracted to the planar difference) and therefore, the measured electrical potential must continue to decrease - this results in negative values for $\Delta V_{M N}$. The opposite is true for Figure 11(b) where a more resistive planar difference effectively creates a lower electrical conductivity (i.e., the current is repelled by the planar difference) and $\Delta V_{M N}$ must continue to increase. Much like the result in Figure 1, there is a $x / D$ where the planar difference has no effect on the

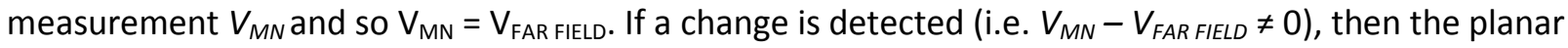
difference has been detected for its position $x / D$. We call this value of $x / D$ the 'look-ahead' distance. For example in Figure 11(a), the condition of $t_{D}, t_{A}=0,15$ yields a look-ahead distance of $x / D \approx 4$. This suggests that this electrode array is capable of identifying a planar difference for $x / D \leq 4$. The lookahead distances observed in these plots are only theoretical; in reality, electrical noise and ground heterogeneity would reduce a realizable look ahead distance.

The figures demonstrate that the $\Delta V_{M N}$ vs. $x / D$ is influenced by the interface region. For example in Figure 11 , the look-ahead distance is greater for the focusing configuration $\left(t_{D}, t_{A}=0,15\right)$ than any of the others, and the configuration with air-filled annulus and disk interface regions $\left(t_{D}, t_{A}=40,15\right)$ exhibits a lesser look-ahead distance consistent with the limited current density propagation shown in Figure 9. For all cases in Figure 11(a), the observed look-ahead distances are considerable and at least $x / D=2$ for all assumptions of the interface region. To place this $20 \mathrm{~m}$ look ahead distance in context, typical ring advances are 1.5-2.0 m and take 30-45 minutes to advance and build. A 20m look ahead distance equates to 10-13 rings and 6-10 hours advance warning.

For all plots in Figures 11 through 14, the condition for hard rock $\left(\sigma_{A}=\sigma_{D}=10^{-15} \mathrm{~S} / \mathrm{m}\right)$ and $\operatorname{EPB}\left(\sigma_{A}=\sigma_{D}=\right.$ $10^{-6} \mathrm{~S} / \mathrm{m}$ ) TBM tunneling shows results that are identical, where plots of $\Delta V_{M N} v s . x / D$ overlap each other. Further, slurry TBM tunneling is not significantly influenced by the interface region. Both of these 
results are consistent with the behavior observed in the current density vs. $x_{\max }$ plots shown in Figures 9 and 10.

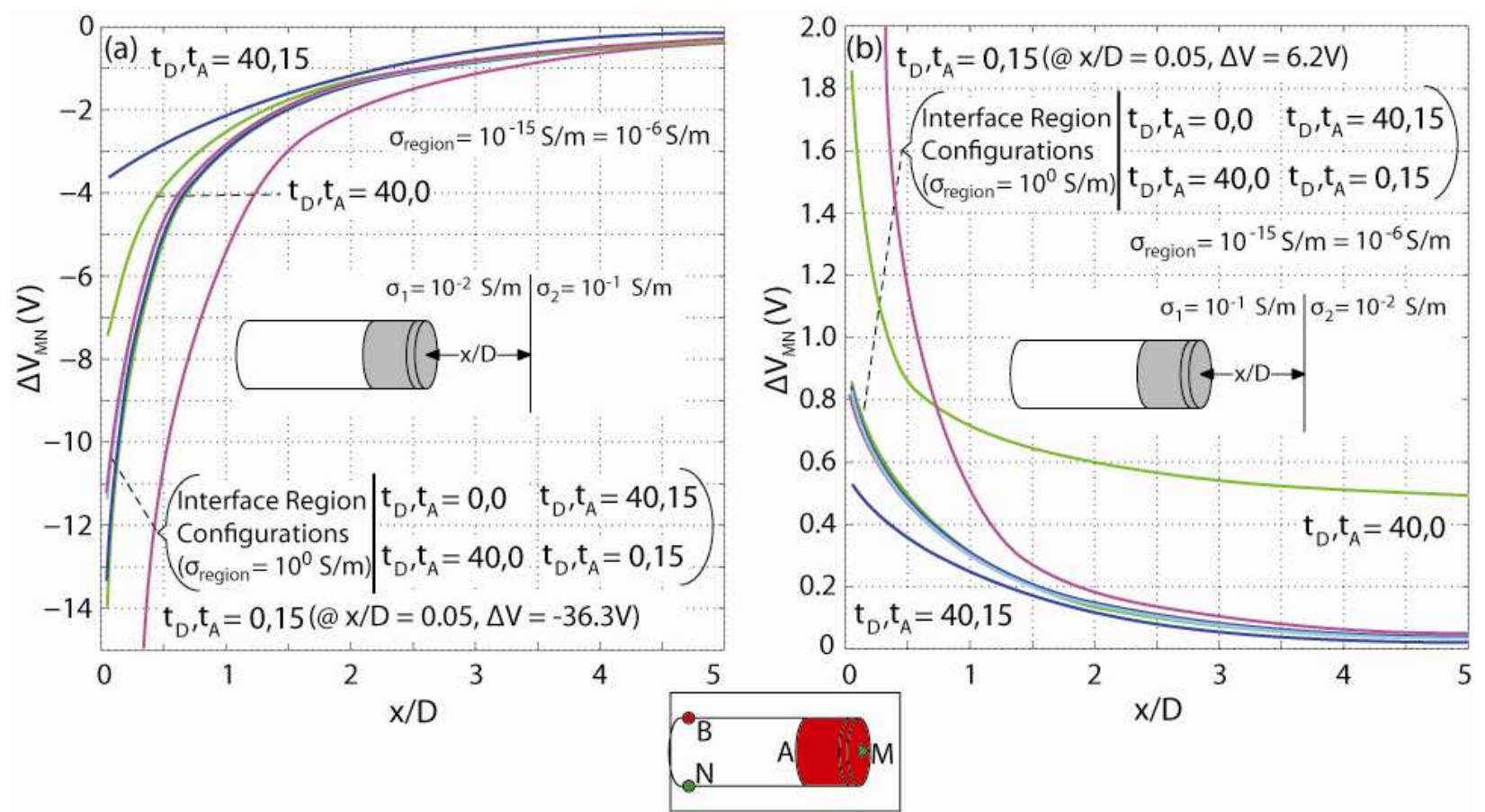

Figure 11: Electrical potential sensitivity to an infinite, vertical planar difference, located at $x / D$, for electrode configuration from Figure 5(a): (a) $\sigma_{1}=10^{-2} \mathrm{~s} / \mathrm{m}$ and $\sigma_{2}=10^{-1} \mathrm{~s} / \mathrm{m}$, (b) $\sigma_{1}=10^{-1} \mathrm{~s} / \mathrm{m}$ and $\sigma_{2}=$ $10^{-2} \mathrm{~S} / \mathrm{m}$.

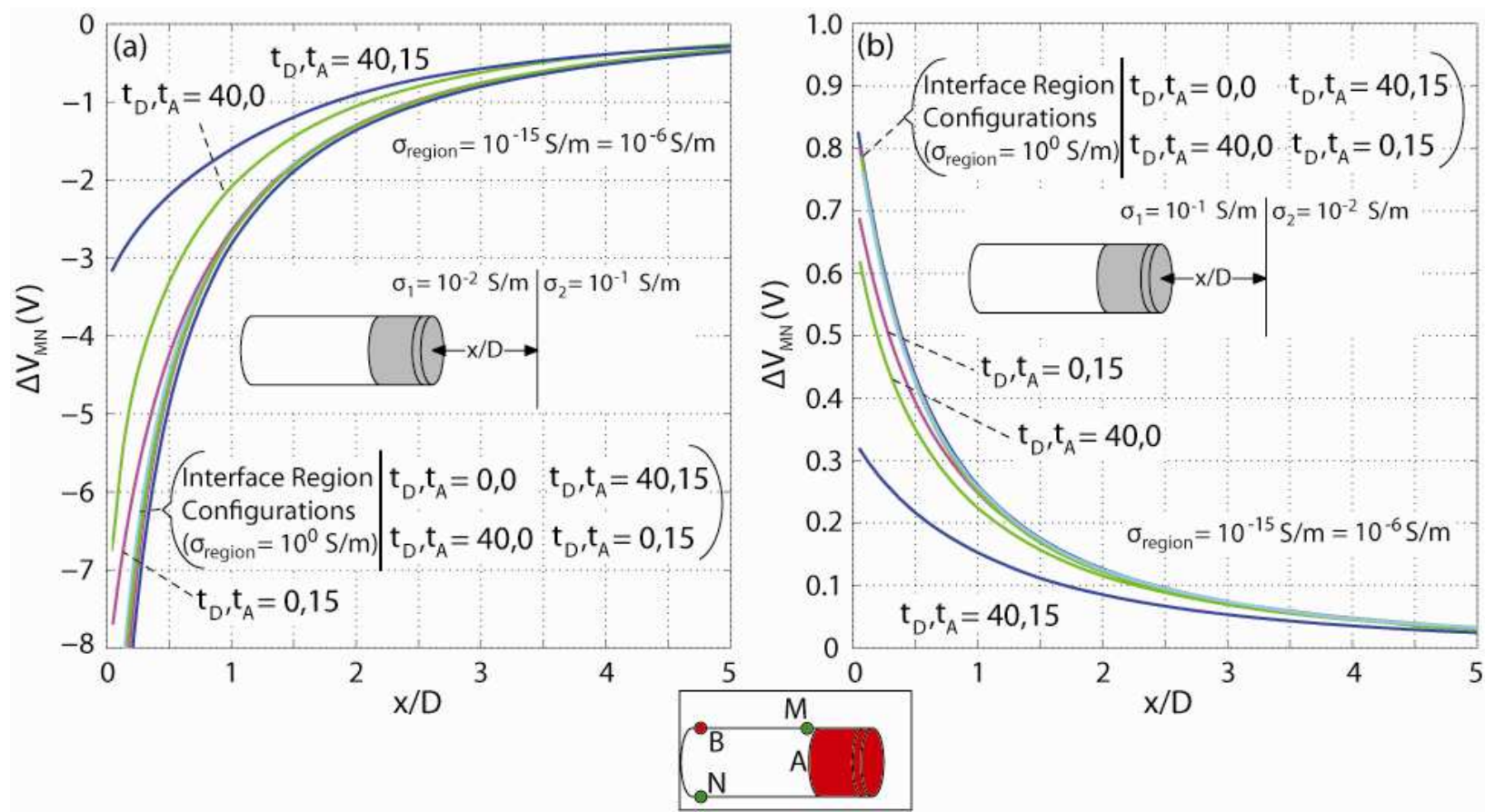


Figure 12: Electrical potential sensitivity to an infinite, vertical planar difference, located at $x / D$, for electrode configuration from Figure $5(\mathrm{c})$ : (a) $\sigma_{1}=10^{-2} \mathrm{~S} / \mathrm{m}$ and $\sigma_{2}=10^{-1} \mathrm{~S} / \mathrm{m}$, (b) $\sigma_{1}=10^{-1} \mathrm{~S} / \mathrm{m}$ and $\sigma_{2}=$ $10^{-2} \mathrm{~S} / \mathrm{m}$.

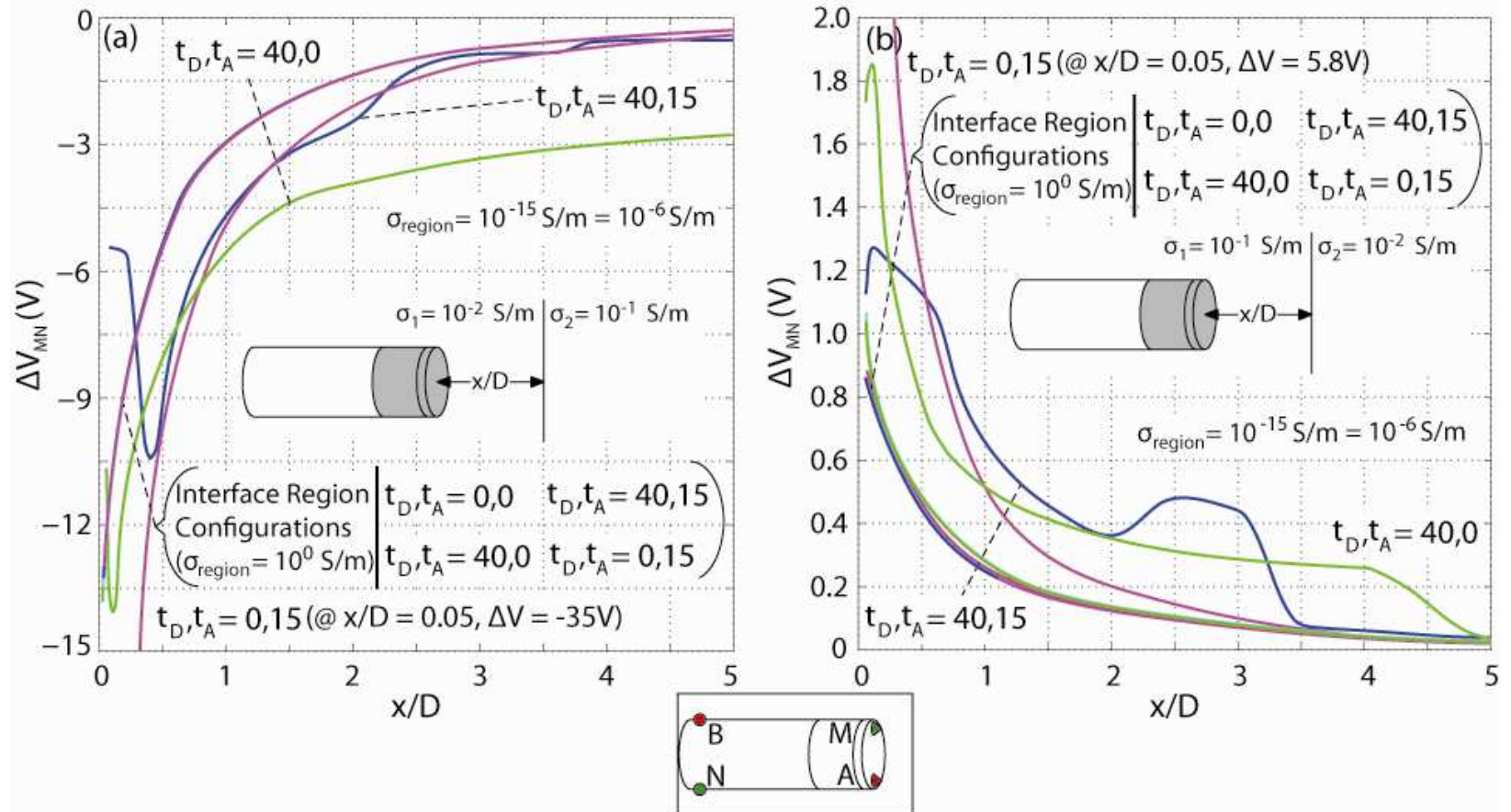

Figure 13: Electrical potential sensitivity to an infinite, vertical planar difference, located at $x / D$, for electrode configuration from Figure 5 (d: (a) $\sigma_{1}=10^{-2} \mathrm{~S} / \mathrm{m}$ and $\sigma_{2}=10^{-1} \mathrm{~S} / \mathrm{m},(\mathrm{b}) \sigma_{1}=10^{-1} \mathrm{~S} / \mathrm{m}$ and $\sigma_{2}=$ $10^{-2} \mathrm{~S} / \mathrm{m}$.

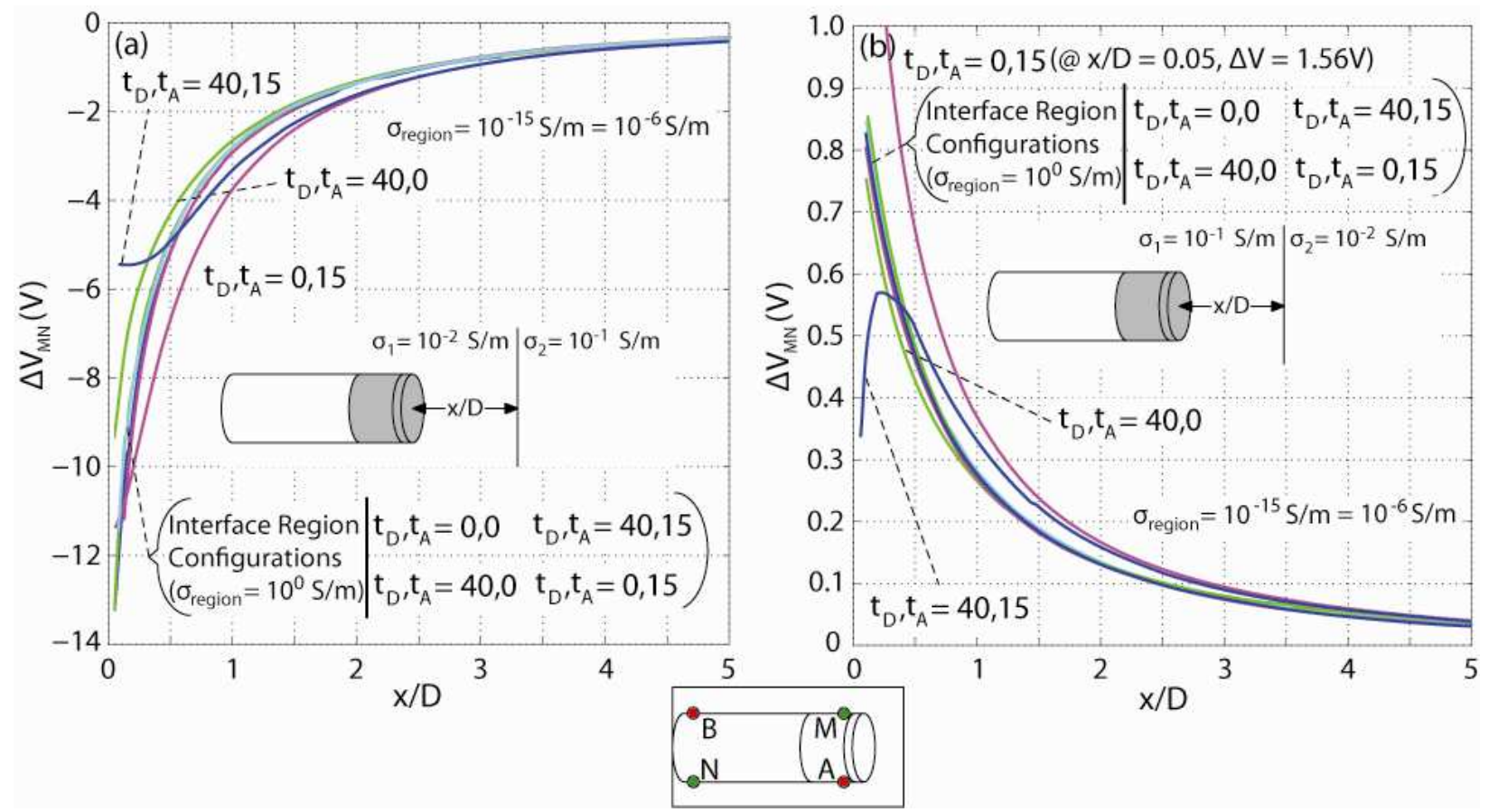


Figure 14: Electrical potential sensitivity to an infinite, vertical planar difference, located at $x / D$, for electrode configuration from Figure 5(f): (a) $\sigma_{1}=10^{-2} \mathrm{~S} / \mathrm{m}$ and $\sigma_{2}=10^{-1} \mathrm{~S} / \mathrm{m},(\mathrm{b}) \sigma_{1}=10^{-1} \mathrm{~S} / \mathrm{m}$ and $\sigma_{2}=$ $10^{-2} \mathrm{~S} / \mathrm{m}$.

In Figures 11 through 14, only the electrode configurations that positioned the current sink behind the TBM $(x<0)$ were shown. For Figures 15 and 16, electrode configurations that position the current sink in front of the TBM $(x>0)$ are shown. Subplots (c) and (d) are zoomed-in plots of (a) and (b), respectively. For smaller $x / D$ values, subplots (c) and (d) are visually similar to those presented in Figures 11 through 14. As $x / D$ decreases, $\Delta V_{M N}$ intensifies. However, subplots (a) and (b) reveal an intermediate region for values of $x / D$ where $\Delta V_{M N}$ remains constant at a magnitude that is non-zero. As $x / D$ increases to a value of $24, \Delta V_{M N}$ approaches zero.

This behavior can be explained by the flow of current through the model and is qualitatively explained by the current flow lines shown by the electrode configurations in Figure 5. Methods which position the current sink behind the TBM, draw current behind the TBM after it escapes into the formation. This causes there to be near zero current densities at significant distances in front of the TBM (e.g. $x>30 \mathrm{~m}$ for Figure 8(a)). When the planar difference crosses into a region that holds significant current density, a non-zero $\Delta V_{M N}$ is recorded. For the cases where the sink is located behind the TBM, there is only one region of significant current density in front of the TBM $(x>0)$. This region is shown the Figure 8 plots. When the current sink is positioned in front of the TBM, all of the current is forced to flow in front of the TBM and to the sink at the surface, thereby creating two concentrated regions of current density: 1 ) directly in front of the TBM and 2) far away at the current sink. These two concentrated regions produce the two regions of changing $\Delta V_{M N}$ that can be observed in Figures 15 and 16.

For Figure 15 and 16, the look-ahead distance is more straight-forward to identify in subplots (c) and (d) but there may be difficulties in the field when the location of planar difference is uncertain (i.e. TBM operator does not know if the planar difference is located close to the sink or if it is close to the TBM). For this paper, the look ahead distance is determined by using the changing $\Delta V_{M N}$ region that is closest to the TBM as shown in subplots (a) and (b). The changing $\Delta V_{M N}$ region nearest the current sink should be considered a false positive for detection and should be disregarded. 

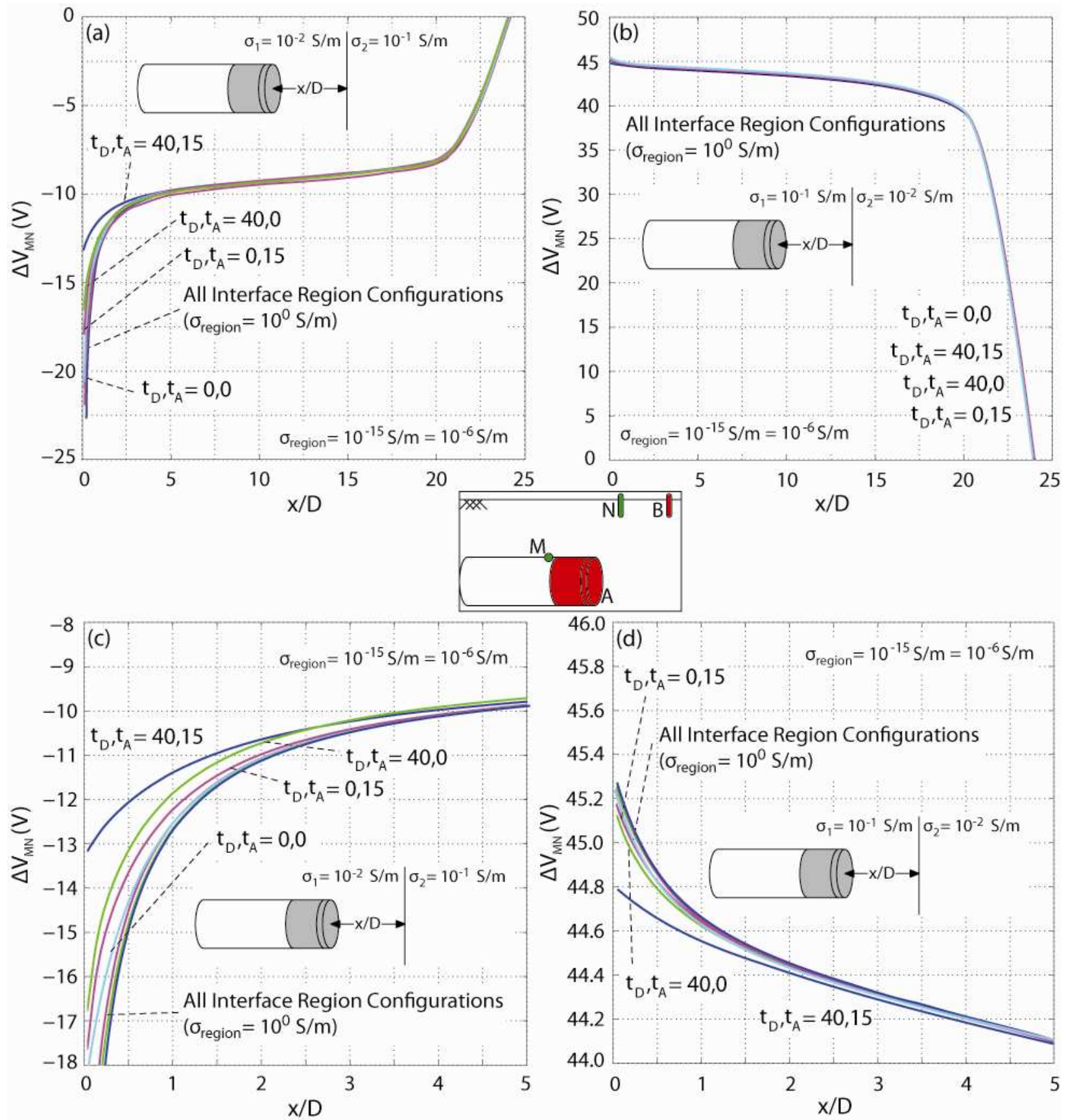

Figure 15: Electrical potential sensitivity to an infinite, vertical planar difference, located at $x / D$, for electrode configuration from Figure $5(\mathrm{~b}):$ (a) $\sigma_{1}=10^{-2} \mathrm{~S} / \mathrm{m}$ and $\sigma_{2}=10^{-1} \mathrm{~S} / \mathrm{m}$, (b) $\sigma_{1}=10^{-1} \mathrm{~S} / \mathrm{m}$ and $\sigma_{2}=$ $10^{-2} \mathrm{~S} / \mathrm{m}$. 

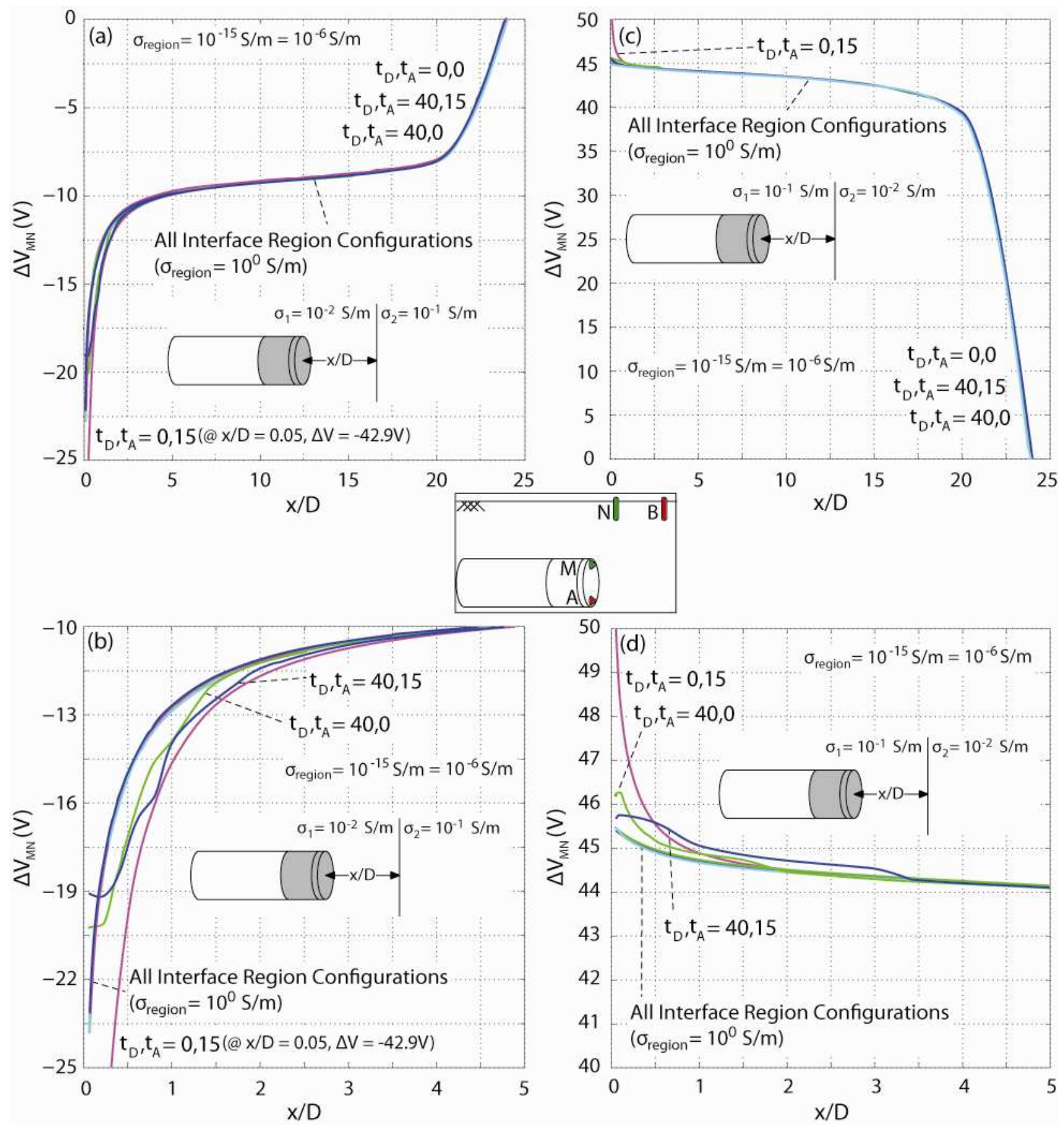

Figure 16: Electrical potential sensitivity to an infinite, vertical planar difference, located at $x / D$, for electrode configuration from Figure 5(f): (a) $\sigma_{1}=10^{-2} \mathrm{~s} / \mathrm{m}$ and $\sigma_{2}=10^{-1} \mathrm{~S} / \mathrm{m}$, (b) $\sigma_{1}=10^{-1} \mathrm{~s} / \mathrm{m}$ and $\sigma_{2}=$ $10^{-2} \mathrm{~S} / \mathrm{m}$.

The previous $\Delta V_{M N}$ vs. $x / D$ plots present a large amount of information and it is difficult to point to the best combination of electrode array, interface region assumption, and TBM type. Tables 1 and 2 summarize look-ahead distances for each combination. Look-ahead distances were determined based upon the slope of each sensitivity curve above. An arbitrary slope $d(\Delta V) / d(x / D) \geq 0.2$ was used. 
Table 1: Estimated look-ahead distances for each Figure 5 electrode configuration and each assumption for the interface region. Ground condition in front of TBM shown for $\sigma_{1}=10^{-2} \mathrm{~S} / \mathrm{m}$ and $\sigma_{2}$ $=10^{-1} \mathrm{~S} / \mathrm{m}$ from Figure 6 . Distances shown in terms of dimensionless distance in front of TBM, $x / D$, in

TBM diameters.

\begin{tabular}{|c|c|c|c|c|c|c|c|}
\hline & & \multicolumn{6}{|c|}{ Look-Ahead Distance (Diamaters of TBM) } \\
\hline \multicolumn{2}{|c|}{$\begin{array}{c}\text { Electrode } \\
\text { Configuration }\end{array}$} & A & 急 & ${ }_{0}^{\mathrm{B}} \quad\left(\begin{array}{l}\mathrm{M} \\
\mathrm{A}\end{array}\right.$ & ${ }_{0}^{\mathrm{N}}$ & 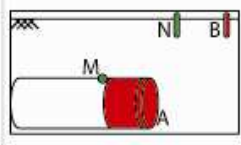 & 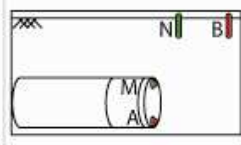 \\
\hline \multicolumn{2}{|c|}{ Hard Rock } & \multicolumn{6}{|c|}{$\sigma_{D}=\sigma_{A}=10^{-15} \mathrm{~S} / \mathrm{m}$} \\
\hline \multirow{4}{*}{$\frac{5}{8}$} & $0 / 0$ & 3.50 & 3.50 & 3.75 & 3.75 & 4.00 & 4.50 \\
\hline & $40 / 15$ & 1.50 & 3.00 & 5.00 & 4.00 & 3.50 & 5.00 \\
\hline & $40 / 0$ & 3.50 & 3.50 & 4.00 & 3.75 & 4.00 & 4.50 \\
\hline & $0 / 15$ & 4.00 & 4.00 & 4.00 & 4.00 & 4.00 & 5.00 \\
\hline \multicolumn{2}{|c|}{$E P B$} & \multicolumn{6}{|c|}{$\sigma_{0}=\sigma_{A}=10^{6} \mathrm{~S} / \mathrm{m}$} \\
\hline \multirow{4}{*}{$\frac{5}{5}$} & $0 / 0$ & 3.50 & 3.50 & 3.75 & 3.75 & 4.00 & 4.50 \\
\hline & $40 / 15$ & 1.50 & 3.00 & 5.00 & 4.00 & 3.50 & 5.00 \\
\hline & $40 / 0$ & 3.50 & 3.50 & 4.00 & 3.75 & 4.00 & 4.50 \\
\hline & $0 / 15$ & 4.00 & 4.00 & 4.00 & 4.00 & 4.00 & 5.00 \\
\hline \multicolumn{2}{|c|}{ Slurry } & \multicolumn{6}{|c|}{$\sigma_{0}=\sigma_{A}=10^{0} \mathrm{~S} / \mathrm{m}$} \\
\hline \multirow{4}{*}{$\stackrel{5}{\$}$} & $0 / 0$ & 3.50 & 3.50 & 3.75 & 3.75 & 4.00 & 4.50 \\
\hline & $40 / 15$ & 3.50 & 3.50 & 3.75 & 3.75 & 4.00 & 4.50 \\
\hline & $40 / 0$ & 3.50 & 3.50 & 3.75 & 3.75 & 4.00 & 4.50 \\
\hline & $0 / 15$ & 3.50 & 3.50 & 3.75 & 3.75 & 4.00 & 4.50 \\
\hline
\end{tabular}

Table 2: Estimated look-ahead distances for each Figure 5 electrode configuration and each assumption for the interface region. Ground condition in front of TBM shown for $\sigma_{1}=10^{-1} \mathrm{~S} / \mathrm{m}$ and $\sigma_{2}$ $=10^{-2} \mathrm{~S} / \mathrm{m}$ from Figure 6. Distances shown in terms of dimensionless distance in front of TBM, $X / D$, in TBM diameters.

\begin{tabular}{|c|c|c|c|c|c|c|c|}
\hline & & \multicolumn{6}{|c|}{ Look-Ahead Distance (Diamaters of TBM) } \\
\hline \multicolumn{2}{|c|}{$\begin{array}{c}\text { Electrode } \\
\text { Configuration }\end{array}$} & 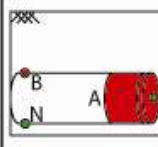 & $\begin{array}{lll}\times & & \\
& M \\
B & A \\
N & A \\
\end{array}$ & 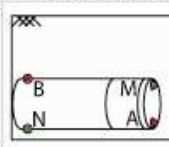 & $\mathrm{C}_{\mathrm{B}}^{\mathrm{B}}$ & 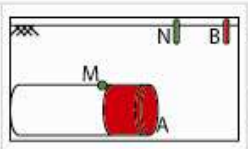 & 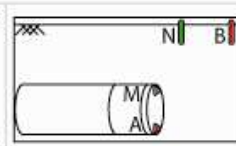 \\
\hline \multicolumn{2}{|c|}{ Hard Rock } & \multicolumn{6}{|c|}{$\sigma_{0}=\sigma_{A}=10^{-15} \mathrm{~s} / \mathrm{m}$} \\
\hline \multirow{4}{*}{$\stackrel{5}{9}$} & $0 / 0$ & 1.00 & 1.00 & 1.00 & 1.00 & 1.00 & 1.50 \\
\hline & $40 / 15$ & 1.00 & 0.25 & 1.50 & 1.00 & 0.75 & 2.00 \\
\hline & $40 / 0$ & 1.00 & 0.75 & 1.00 & 1.00 & 1.00 & 1.50 \\
\hline & $0 / 15$ & 1.50 & 1.00 & 1.50 & 1.25 & 1.00 & 2.00 \\
\hline \multicolumn{2}{|c|}{$E P B$} & \multicolumn{6}{|c|}{$\sigma_{0}=\sigma_{\mathrm{A}}=10^{-6} \mathrm{~S} / \mathrm{m}$} \\
\hline \multirow{4}{*}{$\frac{5}{8}$} & $0 / 0$ & 1.00 & 1.00 & 1.00 & 1.00 & 1.00 & 1.50 \\
\hline & $40 / 15$ & 1.00 & 0.25 & 1.50 & 1.00 & 0.75 & 2.00 \\
\hline & $40 / 0$ & 1.00 & 0.75 & 1.00 & 1.00 & 1.00 & 1.50 \\
\hline & $0 / 15$ & 1.50 & 1.00 & 1.50 & 1.25 & 1.00 & 2.00 \\
\hline \multicolumn{2}{|c|}{ Slurry } & \multicolumn{6}{|c|}{$\sigma_{D}=\sigma_{A}=10^{0} \mathrm{~S} / \mathrm{m}$} \\
\hline \multirow{4}{*}{$\frac{5}{s}$} & $0 / 0$ & 1.00 & 1.00 & 1.00 & 1.00 & 1.00 & 1.50 \\
\hline & $40 / 15$ & 1.00 & 1.00 & 1.00 & 1.00 & 1.00 & 1.50 \\
\hline & $40 / 0$ & 1.00 & 1.00 & 1.00 & 1.00 & 1.00 & 1.50 \\
\hline & $0 / 15$ & 1.00 & 1.00 & 1.00 & 1.00 & 1.00 & 1.50 \\
\hline
\end{tabular}

In general, the cases where the TBM moves toward an incoming conductive difference $\left(\sigma_{1}=10^{-2} \mathrm{~S} / \mathrm{m}\right.$ and $\sigma_{2}=10^{-1} \mathrm{~S} / \mathrm{m}$ ) exhibit the best look-ahead distances. This result is analogous to that for the Figure 2 analysis. Due to the incoming conductive planar difference, more current density is drawn toward the difference and it becomes sensitive at larger distances of $x / D$ than for the more resistive difference. Even though hard rock and EPB tunneling show greater look-ahead distances on average compared to 
slurry tunneling, slurry tunneling shows no influence on look-ahead distance for the interface region and may be the more reliable choice for TBM-integrated-electrical resistivity.

\subsection{Limitations and Implementation Issues}

This study investigated only two combinations of electrical conductivity for the formation around the $\operatorname{TBM}\left(\sigma_{1}\right)$ and the vertical planar difference $\left(\sigma_{2}\right)$. In reality, a wide range of electrical conductivities could be present in much more heterogeneous formation. While the general trends presented above remain true, the levels of current density and potential measurement will change with electrical conductivities. Noise is an important consideration for electrical resistivity methods. It is not yet clear what the electrical noise will look like in the field. Electrical resistivity is only sensitive to an incoming difference if $\Delta V_{M N}$ is greater than the electrical noise - otherwise the difference will go undetected.

This study suggests that the interface region has a significant influence on electrical resistivity measurements for some of the electrode configurations. Selecting an electrode configuration that is not highly influenced by the interface region could be ideal. For example, point current source is relatively unaffected by the interface region configuration compared to full TBM cutterhead and shield injection. Further, slurry tunneling $\left(\sigma_{A}=\sigma_{D}=10^{\circ} \mathrm{S} / \mathrm{m}\right)$ is not influenced by the interface region for any electrode configuration or interface region assumption.

Even though some electrode configurations work well to sense an incoming planar difference, they may be infeasible to implement in the field. For example, configurations that position the current sink and potential electrode $\mathrm{N}$ at the surface may be difficult because these electrodes would have to be connected to a central measurement system (likely in the tunnel). This would require substantial lengths of wire, especially for longer excavations, and may be too difficult in dense surface environments. Also, electrode configurations that use isolated cutting tools for current injection and/or electrical potential measurement require two key components, electrical isolation from the cutterhead and electrical power in the rotating cutterhead.

More recent research has demonstrated that the non-uniqueness of electrical resistivity can be improved by characterizing a material's complex conductivity over a broad range in frequencies with induced polarization (IP) methods (Marshall and Madden 1959, Vinegar and Waxman 1984, Ward 1990, Lesmes and Frye 2001, Binley et al. 2005, Leroy and Revil 2009, Revil and Florsch 2010). Even though IP methods were outside of the scope of this study and paper, the general trends observed in this study should translate well to IP. The investigation of IP methods applied to the TBM tunneling environment will be the focus of a future study.

\subsection{Conclusions}

This paper presents finite element results from a three dimensional study on electrical resistivity methods applied to the TBM tunneling environment. We focus on identifying the electrical influence of a volume that interfaces the TBM to the virgin formation, called the interface region. There is a degree of uncertainty regarding the interface region regarding its electrical conductivity and geometry and so this study considers a range in each. Six electrode arrays are considered that are unique to TBM-integratedelectrical resistivity in an attempt to understand the influence of the interface region to a number of possible implementations in the field. The following conclusions can be drawn from the findings: 
- For all six electrode configurations, significant current densities $\left(>1 \mathrm{~mA} / \mathrm{m}^{2}\right)$ can be observed out to $30 \mathrm{~m}$ ahead of the $10 \mathrm{~m}$ diameter cutting face when the TBM is perfectly coupled to formation (i.e. no interface region). When the interface region is introduced, it has a varying amount of influence on the ability of current density to propagate in front of the TBM. The influence of the interface region is dependent upon the method of the current injection (i.e. full TBM body or isolated cutting tool) as well as the TBM type (i.e. hard rock, EPB and slurry).

- Electrode configurations that inject current from the entire TBM body (cutterhead and shield) are negatively influenced by the presence of the interface region because it isolates the injection electrode from the formation. Current density propagation distance is decreased by as much as $30 \%$ depending upon the configuration of the interface region. The exception to this is when the interface region consists only of the annulus, in which case current is focused through the cutterhead and current density propagation is increased by approximately $20 \%$.

- Electrode configurations that inject current from a single isolated cutting tool are positively influenced by the interface region where current density propagation is increased by approximately $20 \%$. The best case scenario for these electrode configurations is given when the TBM is completely encapsulated by the interface region, effectively shielding the large metal structure from the formation. In contrast to full TBM body injection, cutting tool injection demonstrates less of a range in influence to the interface region.

- Hard rock and EPB tunneling conditions offer identical observations for both current density propagation ahead of the TBM as well as $\Delta V_{M N}$ measurements taken for an incoming vertical planar difference.

- For slurry tunneling, the interface region has near zero influence on either current propagation ahead of the TBM or $\Delta V_{M N}$ measurements taken for an incoming vertical planar difference. This result is advantageous as the interface region is not a variable in electrical resistivity analysis throughout the excavation of the tunnel.

- Electrode arrays that position a current sink on the surface, in front of the TBM, show two regions of sensitivity due to the concentration of current density around the sink. In the field, these electrode arrays may be infeasible to implement and this result may provide a false positive detection of a change ahead of the cutting face.

- Electrical resistivity is more sensitive to an incoming vertical planar difference that is more conductive than for one that is more resistive than the formation surrounding the TBM.

- Simulations suggest that TBM-integrated-electrical resistivity can detect a planar difference in geology up to five diameters in front of the TBM. This look-ahead distance is subject to change for other types of geologic/man-made differences ahead of the TBM due to their contrasting size, location and electrical properties.

\subsection{Acknowledgements}

We thank NSF for funding the SmartGeo Educational Program (Project IGERT: Intelligent Geosystems; DGE-0801692). The opinions expressed in this paper are those of the authors and not the NSF.

\subsection{References}

Atekwana, Estella A., William A. Sauck, and Douglas D. Werkema. "Investigations of geoelectrical signatures at a hydrocarbon contaminated site."Journal of Applied Geophysics 44.2 (2000): 167-180. 
Atekwana, Estella A., et al. "Geophysical investigation of vadose zone conductivity anomalies at a hydrocarbon contaminated site: implications for the assessment of intrinsic bioremediation." Journal of Environmental \& Engineering Geophysics 7.3 (2002): 103-110.

Benson, Alvin K., Kelly L. Payne, and Melissa A. Stubben. "Mapping groundwater contamination using dc resistivity and VLF geophysical methods-A case study." Geophysics 62.1 (1997): 80-86.

Binley, Andrew, and Andreas Kemna. "DC resistivity and induced polarization methods." Hydrogeophysics. Springer Netherlands, 2005. 129-156.

Binley, Andrew, et al. "Relationship between spectral induced polarization and hydraulic properties of saturated and unsaturated sandstone." Water Resources Research 41.12 (2005).

Chang, Soo-Ho, et al. "Performance prediction of TBM disc cutting on granitic rock by the linear cutting test." Tunnelling and Underground Space Technology21.3 (2006): 271.

Frye, Kevin M., David P. Lesmes, and F. Dale Morgan. "The influence of pore fluid chemistry on the induced polarization response of rocks and soils."Proceedings of the Symposium on the Application of Geophysics to Engineering and Environmental Problems. Vol. 77. 1998.

Herman, Rhett. "An introduction to electrical resistivity in geophysics."American Journal of Physics 69.9 (2001): 943-952.

Kaus, Arnim, and Wolf Boening. "BEAM-Geoelectrical Ahead Monitoring for TBM-Drives." Geomechanics and Tunnelling 1.5 (2008): 442-449.

Kirsch, Reinhard, and Ugur Yaramanci. Geoelectrical methods. Springer Berlin Heidelberg, 2009.

Kopp, Thomas. "Real-Time Monitoring of Geological Conditions during Mechanized Tunnelling by Means of BEAM4 Methods." 1st Eastern European Tunnelling Conference. 2012.

Leroy, Philippe, and André Revil. "A mechanistic model for the spectral induced polarization of clay materials." Journal of Geophysical Research: Solid Earth (1978-2012) 114.B10 (2009).

Lesmes, David P., and Kevin M. Frye. "Influence of pore fluid chemistry on the complex conductivity and induced polarization responses of Berea sandstone. "Journal of Geophysical Research: Solid Earth (1978-2012) 106.B3 (2001): 4079-4090.

Marshall, Donald J., and Theodore R. Madden. "Induced polarization, a study of its causes." Geophysics 24.4 (1959): 790-816.

Mazac, O., W. E. Kelly, and I. Landa. "Surface geoelectrics for groundwater pollution and protection studies." Journal of Hydrology 93.3 (1987): 277-294.

Meju, Maxwell A. "Geoelectrical investigation of old/abandoned, covered landfill sites in urban areas: model development with a genetic diagnosis approach."Journal of Applied Geophysics 44.2 (2000): 115150.

Mooney, M. A., M. Karaoulis, and A. Revil. "Investigation of Geoelectric-While-Tunneling Methods through Numerical Modeling." WTC, 2013. 
Revil, A., and Nicolas Florsch. "Determination of permeability from spectral induced polarization in granular media." Geophysical Journal International 181.3 (2010): 1480-1498.

Reynolds, John M. An Introduction to Applied and Environmental Geophysics. Chichester: John Wiley, 2011.

Samouëlian, Anatja, et al. "Electrical resistivity survey in soil science: a review." Soil and Tillage research 83.2 (2005): 173-193.

Schaeffer, K. "Geoelectrics-While-Tunneling: Methodology and Performance Aspects." North American Tunneling: 2014 Proceedings (2014).

Szalai, Sandor, and Laszlo Szarka. "On the classification of surface geoelectric arrays." Geophysical Prospecting 56.2 (2008): 159-175.

Telford, William Murray, and Robert E. Sheriff. Applied geophysics. Vol. 1. Cambridge university press, 1990.

Vinegar, H. J., and M. H. Waxman. "Induced polarization of shaly sands."Geophysics 49.8 (1984): 12671287.

Ward, Stanley H. "Resistivity and induced polarization methods." Geotechnical and environmental geophysics 1 (1990): 147-189.

Xue, Jing, et al. "Soft rock cutting mechanics model of TBM cutter and experimental research." Intelligent Robotics and Applications. Springer Berlin Heidelberg, 2009. 383-391. 